\title{
Higgs boson studies with the ATLAS detector at the Large Hadron Collider
}

\author{
Giovanni Marchiori* \\ on behalf of the ATLAS Collaboration \\ LPNHE Paris \\ E-mail: giovanni.marchiori@lpnhe.in2p3.fr
}

In July 2012 a particle with properties consistent with those of a Higgs boson was discovered by the ATLAS and CMS experiments at the LHC. In this report I summarise the results of the measurements performed by the ATLAS experiment of the properties and the searches for rare and forbidden decays of this particle using the Run 1 LHC data, before concluding with an outlook on the future perspectives.

Proceedings of the Corfu Summer Institute 2015 "School and Workshops on Elementary Particle Physics and Gravity"

1-27 September 2015

Corfu, Greece

${ }^{*}$ Speaker. 


\section{Introduction}

The main aim of the ATLAS experiment (A Toroidal LHC ApparatuS) [1] at the Large Hadron Collider (LHC) [2] is the investigation of physics above the electroweak symmetry-breaking (EWSB) scale, in an energy range previously unexplored. Expected to be located approximately within 100 and $1000 \mathrm{GeV}$, this scale becomes experimentally accessible in the highly energetic collisions of multi- TeV proton beams accelerated inside the LHC.

In the Standard Model (SM) [3, 4, 5, 6, 7] of elementary particles, spontaneous EWSB arises from the presence of a doublet of complex Higgs fields with a non trivial vacuum structure, characterized by a set of degenerate ground states that minimize the Higgs-field potential and have non-zero expectation value, $v=246 \mathrm{GeV}$. As a consequence of EWSB, the $W$ and $Z$ gauge bosons and the fundamental matter particles (quarks and leptons) acquire a mass, which otherwise needs to vanish for the SM lagrangian without the Higgs field to be renormalizable and gauge-invariant. The quantum excitations of the Higgs field near the physical vacuum correspond to a new fundamental, scalar, electrically neutral particle, the Higgs boson $H$, whose couplings to the other particles are predicted to be proportional to the particle masses $[8,9,10,11,12,13]$. The mass of the new boson must be below $1 \mathrm{TeV}$ in order to ensure that the longitudinal $W$ boson scattering amplitude, which otherwise would grow with the center-of-mass (CM) energy $(\sqrt{s})$ squared, does not violate unitarity for $\sqrt{s} \gtrsim 1 \mathrm{TeV}$.

The first evidence of a particle with a mass around $125 \mathrm{GeV}$ and properties consistent with those of a Higgs boson was obtained by the ATLAS [14] and CMS [15] experiments in July 2012 based on the analysis of approximately $5 \mathrm{fb}^{-1}$ of proton-proton $(p p)$ collisions at $\sqrt{s}=7$ and $5 \mathrm{fb}^{-1}$ of $p p$ collisions at $\sqrt{s}=8 \mathrm{TeV}$ produced by the LHC in 2011 and 2012, respectively. After the discovery, the larger dataset collected until the end of 2012, before a 2-year long shutdown, has allowed the two collaborations to perform further studies, including precision measurements on some properties of this particle and searches for decays that are rare or even forbidden in the SM. In this report I will summarise the results of such measurements performed by the ATLAS experiment, before concluding with an outlook on the future perspectives.

\section{Experimental setup}

\subsection{The Large Hadron Collider}

The LHC [2] is a two-ring superconducting hadron accelerator and collider, whose tunnel is located between $45 \mathrm{~m}$ and $170 \mathrm{~m}$ underground, beneath the Franco-Swiss border near Geneva, Switzerland. With a length of $26.7 \mathrm{~km}$, a maximum achieved $p p$ center-of-mass energy $\sqrt{s}=$ $13 \mathrm{TeV}$ (in 2015) and a design value of $\sqrt{s}=14 \mathrm{TeV}$, the LHC is the world's largest and highestenergy particle accelerator.

Bunches of more than 100 billion protons each circulate in opposite directions in the two rings, and collide in four interaction regions. The LHC started to collide protons at the end of 2009 , first at $\sqrt{s}=900 \mathrm{GeV}$, and later at $\sqrt{s}=2.36 \mathrm{TeV}$. In March 2010, after a short winter shutdown, collisions of $3.5 \mathrm{TeV}$ proton beams were successfully established, and then delivered to the experiments throughout the rest of 2010 and 2011, with a short interruption during winter 20102011. The instantaneous peak luminosity was progressively increased from about $10^{27} \mathrm{~cm}^{-2} \mathrm{~s}^{-1}$ to 
$3.65 \times 10^{33} \mathrm{~cm}^{-2} \mathrm{~s}^{-1}$. In 2012 the beam energy was increased to $4 \mathrm{TeV}$ and the peak luminosity, constantly above $10^{33} \mathrm{~cm}^{-2} \mathrm{~s}^{-1}$, rose to $7.7 \times 10^{33} \mathrm{~cm}^{-2} \mathrm{~s}^{-1}$, close to its design value. At the end of February 2013 the LHC was shut down, ending its first data-taking phase ("Run 1"). Almost two years of repair and upgrade activities, concerning both the accelerator and the detector, took place, after which the LHC was successfully restarted ("Run 2") at a center-of-mass energy of $13 \mathrm{TeV}$, close its the design value.

\subsection{The ATLAS detector}

The ATLAS detector [1] is a multi-purpose particle detector with approximately forwardbackward symmetric cylindrical geometry. ${ }^{1}$ With a length of $44 \mathrm{~m}$ and a diameter of $25 \mathrm{~m}$, ATLAS, which weighs approximately 7000 tons, is the LHC detector occupying the largest volume. It consists of several nested sub-detectors, which are described in the following, starting from the innermost one and going outwards.

The inner tracking detector (ID) covers $|\eta|<2.5$ and consists of a silicon pixel detector, a silicon microstrip detector, and a straw-tube transition radiation tracker (TRT). The ID is surrounded by a thin superconducting solenoid providing a $2 \mathrm{~T}$ axial magnetic field and by a high-granularity lead/liquid-argon (LAr) sampling electromagnetic (EM) calorimeter. The EM calorimeter measures the energy and the position of electromagnetic showers with $|\eta|<3$.2. It includes a presampler (for $|\eta|<1.8$ ) and three sampling layers, longitudinal in shower depth, up to $|\eta|=2.5$. The hadronic calorimeter, surrounding the electromagnetic one and covering $|\eta|<4.9$, is a sampling calorimeter which uses either scintillator tiles or LAr as the active medium, and steel, copper or tungsten as the absorber material. The muon spectrometer (MS) surrounds the calorimeters and consists of three large superconducting air-core toroid magnets, each with eight coils, a system of precision tracking chambers $(|\eta|<2.7)$, and fast tracking chambers for triggering $(|\eta|<2.4)$. Precision tracking in the MS volume is provided by three layers of Monitored Drift Tube Chambers (MDT); for $|\eta|>2$, the inner layer is instrumented with a quadruplet of Cathode Strip Chambers (CSC) instead of MDTs. Muon triggering capability is provided by Resistive Plate chambers (RPC) for $|\eta|<1.05$ and Thin Gap Chambers (TGC) for $1.0<|\eta|<2.4$.

A two-level trigger system selects events to be recorded for offline analysis. The first-level trigger is hardware based, while the second, high-level trigger is implemented in software and employs algorithms similar to those used offline to identify lepton and photon candidates.

The ATLAS detector has collected data during stable LHC $p p$ collisions with an average datataking efficiency greater than $93 \%$ in 2010-2012. Only events that pass one of the high-level triggers are kept for analysis. Events with data quality problems are discarded. The integrated luminosity after the trigger and data quality requirements corresponds to about $20 \mathrm{fb}^{-1}$ at $\sqrt{s}=$ $8 \mathrm{TeV}$ and $5 \mathrm{fb}^{-1}$ at $7 \mathrm{TeV}$.

\subsection{Particle reconstruction in ATLAS}

Charged-particle tracks with $p_{\mathrm{T}}>0.5 \mathrm{GeV}$ and within the ID acceptance $(|\eta|<2.5)$ are re-

\footnotetext{
${ }^{1}$ ATLAS uses a right-handed coordinate system with its origin at the nominal interaction point (IP) in the center of the detector and the $z$-axis along the beam pipe. The $x$-axis points from the IP to the center of the LHC ring, and the $y$-axis points upward. Cylindrical coordinates $(r, \phi)$ are used in the transverse plane, $\phi$ being the azimuthal angle around the $z$-axis. The pseudorapidity is defined in terms of the polar angle $\theta$ as $\eta=-\ln \tan (\theta / 2)$.
} 
constructed by the ID with two complementary track-finding algorithms that start from space points in the silicon (pixel and microstrip) detectors and from hits in the TRT [16]. The typical transverse momentum resolution from the simulation is $\frac{\sigma_{p_{\mathrm{T}}}}{p_{\mathrm{T}}}=0.05 \% \frac{p_{\mathrm{T}}}{\mathrm{GeV}} \oplus 1 \%$.

Electron and photon candidates are reconstructed and identified using information from the electromagnetic calorimeter and the inner detector, as described in Refs. [17, 18] and Refs. [19, 20], respectively. Their energies are calibrated from the raw signals in the cells of the electromagnetic calorimeter using the algorithms described in Ref. [21], with a typical resolution $\frac{\sigma_{E}}{E}=\frac{10 \%}{\sqrt{E / \mathrm{GeV}}} \oplus$ $0.7 \%$ in the central part of the calorimeter $(|\eta|<1.37)$ and slightly worse in the more forward region $1.37<|\eta|<2.47$.

Electron candidates in the region $|\eta|<2.47$ are identified by associating charged-particle tracks with deposits of energy in the electromagnetic calorimeters. The large signal induced on the TRT anode wires by transition radiation X-ray photons and the lateral and longitudinal development of the electromagnetic shower in the calorimeter allow electron/pion discrimination. The TRT information is combined with shower shape variables of the electromagnetic calorimeter, leakage in the hadronic calorimeter, track quality, track-cluster distance and $E / p$ into an algorithm designed to identify prompt electrons with high efficiency and good rejection of background from hadronic jets.

Photon candidates with $|\eta|<2.47$ are identified as deposits of energy in the electromagnetic calorimeter that are either not associated to charged-particle tracks or are matched to tracks consistent with a $\gamma \rightarrow e^{+} e^{-}$conversion. Shower shape variables of the electromagnetic calorimeter and leakage in the hadronic calorimeter are used to distinguish prompt photons from backgrounds from hadronic jets.

Muon candidates are formed starting from tracks reconstructed either in the ID $(|\eta|<2.5)$ or in the MS $(|\eta|<2.7)$ [22]. If a track is reconstructed both in the ID and in the MS, the two independent momentum measurements are combined. In the center of the barrel region $(|\eta|<0.1)$, which lacks MS coverage, ID tracks are identified as muons using the profile of the associated energy deposits in the calorimeter. Quality requirements on the number of hits in the ID and/or in the MS and on the agreement between the momentum measurement of the two subdetectors (in case a track is reconstructed by both) are used to suppress background muons coming mainly from pion and kaon decays, as well as hadrons reconstructed as muons. The muon momentum resolution $\sigma_{p_{\mathrm{T}}} / p_{\mathrm{T}}$ increases from around $3 \%$ for $p_{\mathrm{T}}=20 \mathrm{GeV}$ to $\approx 10 \%$ at $p_{\mathrm{T}}=1 \mathrm{TeV}$.

Jets with $|\eta|<4.5$ are reconstructed from three-dimensional, noise-suppressed topological clusters of calorimeter cells, using the infrared- and collinear-safe anti- $k_{t}$ algorithm [23] with radius parameter $\Delta R=\sqrt{(\Delta \eta)^{2}+(\Delta \phi)^{2}}=0.4$. The topological clusters are formed by grouping together cells that have significant energies compared to the expected noise and are adjacent with each other either. The jet four-momenta are constructed from a sum over their constituent cells, treating each as an $(E, \vec{p})$ four-vector with zero mass, and are then recalibrated [24, 25, 26] to account for instrumental effects, such as inactive material and non-compensation, as well as for the additional energy due to pile-up. The jet energy resolution, $\frac{\sigma_{E}}{E}=\frac{a}{\sqrt{E}} \oplus \frac{b}{E} \oplus c$, has a sampling term $a$ of about $60 \%$ in the central pseudorapidity region $(|\eta|<1.5)$ and about $100 \%$ in the more forward regions; the constant term $c$ is around $2-3 \%$ for $|\eta|<2.5$ and up to $10 \%$ for $|\eta|>2.5$, while the noise term $b$ is of the order of a few GeV. Quality criteria based on the jet timing information and 
on the fraction of jet energy in the electromagnetic and hadronic calorimeters, as well as on the scalar sum of the transverse momenta of the ID tracks matched to the jet, are applied to suppress fake jets from calorimeter noise, cosmic rays and beam-related backgrounds. For jets in the ID acceptance $(|\eta|<2.5)$, the fraction (jet vertex fraction, JVF) of the scalar sum of the $p_{\mathrm{T}}$ of the tracks, associated with the jet and matched to the selected primary vertex, with respect to the scalar sum of the $p_{\mathrm{T}}$ of the tracks associated with the jet is sometimes required to be greater than a certain threshold $(0.25-0.75)$ to reduce the number of pile-up jets. Jets from $b$-quarks ( $b$-jets) in the ID acceptance $(|\eta|<2.5)$ are identified by means of multivariate algorithms [27] that use as input quantities like track impact parameters and the presence of displaced secondary vertices to separate $b$-jets, $c$-jets and light jets (from light quarks and gluons). For a $b$-jet efficiency of $80 \%$ the typical rejection factors are 26 and 1400 for $c$-jets and light jets, respectively.

The missing transverse energy $E_{\mathrm{T}}^{\text {miss }}$ is reconstructed from energy deposits in the calorimeters and from muon tracks (since muons deposit only small amounts of energy in the calorimeters) $[28,29]$. For each calorimeter cell belonging to three-dimensional, noise-suppressed topological clusters, the transverse energy vector $\vec{E}_{\mathrm{T}}=E \sin \theta \hat{n}$ is computed, where $E$ is the measured energy, $\theta$ is the polar angle, and $\hat{n}$ is a unit vector, in the transverse plane, pointing from the beam axis to the cell. $E_{\mathrm{T}}^{\text {miss }}$ is the magnitude of the vector opposite to the sum of the $\vec{E}_{\mathrm{T}}$ vectors measured in the calorimeter and of the transverse momenta $\vec{p}_{\mathrm{T}}$ of the reconstructed muon tracks. The calorimeter cell energies are calibrated according to the particle type (photon, electron, hadrons, ..) assigned to the cluster they belong to. As a consequence of momentum conservation and of the quasi-hermeticity of the ATLAS detector, a large missing transverse energy is an indication of high- $p_{\mathrm{T}}$ weakly-interacting particles (like neutrinos) produced in the $p p$ collisions. $^{2}$

Leptonic decays of the $\tau$ leptons are identified by the presence of an electron or a muon and missing transverse energy. Hadronic $\tau$ decays are reconstructed as jets that are matched to one or three tracks with $p_{\mathrm{T}}>1 \mathrm{GeV}$ and are within a cone of radius $R=0.2$ around the jet axis [30]. The total charge of the three tracks must be equal to \pm 1 . Background from hadronic jets is suppressed through a multivariate algorithm that uses as inputs the tracks with $p_{\mathrm{T}}>1 \mathrm{GeV}$ and the energy deposited in the calorimeter in the two regions $\Delta R<0.2$ and $0.2<\Delta R<0.4$ with respect to the jet axis.

\section{SM Higgs boson production and decays at the LHC}

In the SM, the Higgs boson is a scalar particle with even parity $\left(J^{P C}=0^{++}\right)$. Its couplings to the other SM particles are determined from the particle masses and the vacuum expectation value of the Higgs field, $v \approx 246 \mathrm{GeV}$ (as measured from muon decays). The Higgs boson couplings are proportional to their masses $m_{f}, g_{H f f}=\frac{m_{f}}{v}$; for vector bosons $V(V=W, Z)$ of mass $m_{V}$, the couplings are proportional to the masses squared: $g_{H V V}=\frac{2 m_{V}^{2}}{v}$. The Higgs boson mass itself, $m_{H}$ (and thus also its self-coupling $\lambda$ ) is not predicted by the SM; however, since the couplings are known, once the mass is fixed all the production and decay properties (cross sections, branching ratios, total width) of the Higgs boson are known since all the partial widths to any $f \bar{f}$ or $V V$ state can be calculated.

\footnotetext{
${ }^{2}$ as the initial longitudinal momentum of the interacting partons is unknown, only the conservation ot momentum in the transverse plane is exploited.
} 
The Higgs boson production cross section at $\sqrt{s}=8 \mathrm{TeV}$ and the Higgs boson branching ratios for different decay channels are presented as a function of $m_{H}$ in Fig. 1.
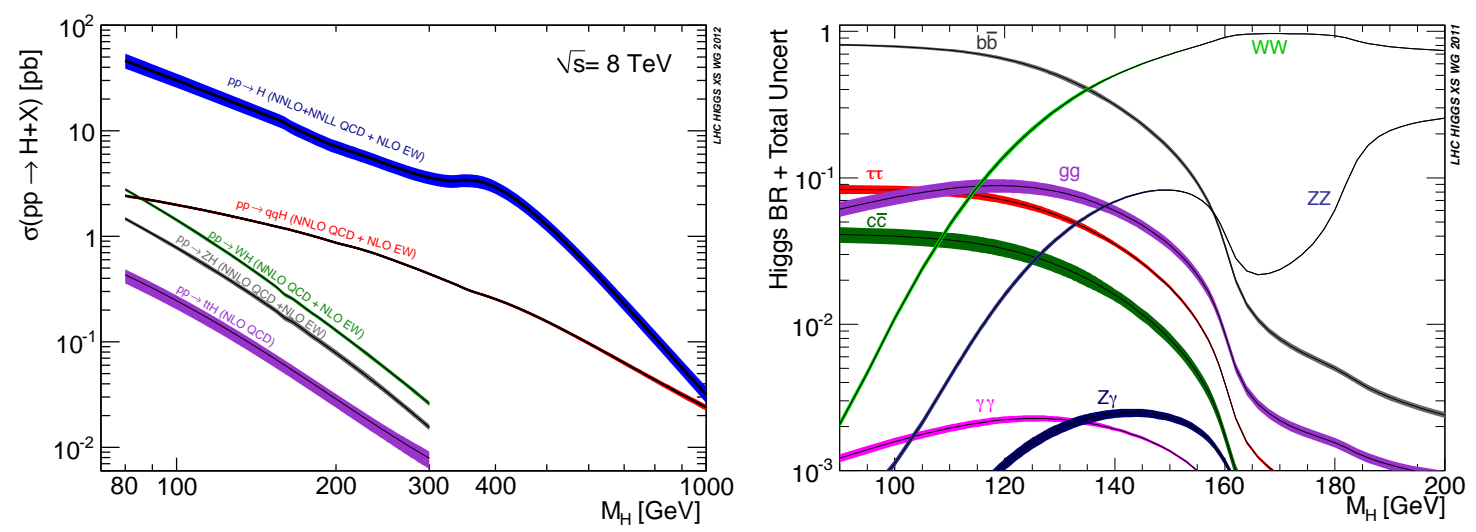

Figure 1: Left: SM Higgs boson production cross section vs Higgs boson mass at $\sqrt{s}=8 \mathrm{TeV}$, for different production mechanisms: gluon fusion $(p p \rightarrow H)$, VBF $(p p \rightarrow q q H)$, associated production with a vector boson $(p p \rightarrow W H, p p \rightarrow Z H)$ and associated production with a $t \bar{t}$ pair $(p p \rightarrow t t H)$. Right: SM Higgs boson branching ratios vs Higgs boson mass, for various final states. Taken from Refs. [31, 32].

At $\sqrt{s}=8 \mathrm{TeV}$ the production cross section for a Higgs boson with a mass of $125 \mathrm{GeV}$ is $\sigma_{H}=22.3 \pm 2.0 \mathrm{pb}$. The cross section at $\sqrt{s}=7 \mathrm{TeV}$ is about $25 \%$ lower; at the design CM energy of $14 \mathrm{TeV}$ it is $\approx 2.6$ higher. Such values of the cross sections imply that if the SM Higgs boson exists and has a mass $m_{H}=125 \mathrm{GeV}$, about half million of them should have been produced in the interaction point of the ATLAS detector by the $p p$ collisions of the LHC Run 1. The number of those that would be reconstructed however is much less, as will be shown in the following, due to the detector acceptance and the efficiencies of the reconstruction and selection criteria.

The main Higgs boson production mode is gluon fusion $(\mathrm{ggF})$, contributing to $86 \%$ of the total cross section, followed by vector-boson fusion (VBF: 7\%), associated production with a vector boson (VH: 5\%), and associated production of a $b \bar{b}$ or $t \bar{t}$ pair $(b b H, t t H: 1.5 \%)$. The different production processes are sensitive to different Higgs boson couplings:

- gluon fusion and $t t H$ or $b b H$ are dominated by amplitudes in which the Higgs boson interacts with two top or two bottom quarks and are thus sensitive to the couplings to two fermions, $t$ and $b$;

- VBF and $V H$ production are induced by amplitudes in which the Higgs boson interacts with a $V V$ pair and are thus sensitive to the couplings to the vector bosons, $W$ and $Z$.

Experimentally, the different production processes are characterised by different signatures; modes that contribute less to the total cross section compared to the dominant mechanism of gluon fusion typically produce additional particles that can be identified with good efficiency and lead to final states that can be selected with better signal-to-background $(S / B)$ ratio. In particular:

- VBF events, in which two quarks from the colliding protons radiate two vector bosons that annihilate into a Higgs boson, are characterized by two additional jets (from the two quarks 
that have radiated the bosons), with large invariant mass and rapidity separation, with little hadronic activity in between;

- in $\mathrm{VH}$ events there is a vector boson that can be identified by means of its decays to leptons or quarks. In the former case one requires either zero leptons (usually electrons or muons, due to their higher identification efficiency and background rejection compared to $\tau$ ) and large $E_{\mathrm{T}}^{\text {miss }}$ from $Z \rightarrow v \bar{v}$ decays $(B R \approx 20 \%)$; one lepton and large $E_{\mathrm{T}}^{\text {miss }}$, with transverse invariant mass near $m_{W}$, for $W \rightarrow \ell v$ decays, $\ell=e, \mu(B R \approx 22 \%)$; or two same-flavour, opposite-sign leptons with invariant mass $m_{\ell \ell} \approx m_{Z}$ for $Z \rightarrow \ell \ell$ decays $(B R \approx 6.7 \%)$. In the latter case a pair of high- $p_{\mathrm{T}}$ jets with invariant mass close to $m_{W}$ or $m_{Z}$ is searched for in order to reconstruct hadronic $W(B R \approx 67 \%)$ and $Z(B R \approx 70 \%)$ boson decays;

- $t t H$ events are characterised by two top quarks that disintegrate promptly to $W b W b$, with the $W$ bosons then decaying either leptonically or hadronically. The events are thus characterised by: a large number of jets, from the top and the hadronic $W$ decays; $b$-jets, from the top decays; leptons and $E_{\mathrm{T}}^{\text {miss }}$ from the leptonic $W$ decays.

For $m_{H}=125$ the total width of the SM Higgs boson is small $\left(\Gamma_{H} \approx 4.1 \mathrm{MeV}\right)$ compared to the experimental resolution in any of the final states that are produced. At such a mass, many Higgs boson decay channels are kinematically allowed and have non-negligible branching ratios.

The main decay mode is the disintegration into a $b \bar{b}$ pair $(B R \approx 57 \%)$. Its reconstruction in gluon fusion events, however, is complicated by the overwhelming background from QCD production of multi-jet events. For this reason, $H \rightarrow b \bar{b}$ decays are mostly searched for in the "cleaner" $V H$ production mode, where the vector boson decays leptonically. Such events constitute only about $0.6 \%$ of the events in which a Higgs boson is produced.

Decays to other pairs of quarks $(c \bar{c})$ or gluons $(g g)$ have large $B R(\approx 3 \%$ and $\approx 9 \%$, respectively) but can't be easily discriminated (due to the absence of a dedicated charm jet tagging) and are affected by larger backgrounds compared to $b \bar{b}$ (since the $Z+$ light jet cross section is larger than that of $Z+\mathrm{b}$-jets as a consequence of the parton distribution functions of the colliding protons).

Decays to a pair of vector bosons have $B R \approx 22 \%\left(H \rightarrow W W^{*}\right)$ and $B R \approx 2.7 \%\left(H \rightarrow Z Z^{*}\right)$. However, the final states that have the best sensitivity are those in which both vector bosons decay leptonically, $H \rightarrow Z Z^{*} \rightarrow 4 \ell$ and $H \rightarrow W W^{*} \rightarrow \ell v \ell v$, that correspond to only approximately $0.01 \%$ and $1 \%$ of all the Higgs bosons initially produced. Other bosonic decays produce final states with photons: $H \rightarrow \gamma \gamma(B R=0.22 \%)$ and $H \rightarrow Z \gamma(B R=0.15 \%)$. The latter, however, is reconstructed in the $Z \rightarrow \ell \ell$ decay channel to suppress background from QCD $\gamma+$ jet events; only approximately $0.01 \%$ of all the Higgs bosons that are produced decay to such final state.

Among the leptonic decays, the one with the largest branching ratio is that to $\tau$ pairs $(B R=$ 6.3\%). Decays to $\mu \mu$ or $e e$ final states, which have better invariant mass resolution due to the absence of neutrinos and the good resolution of the EM calorimeter and of the tracker, and suffer from less backgrounds, have much smaller branching ratios due to the hierarchy of the lepton masses and the proportionality of the Higgs boson couplings to $m_{f}$ : for instance, for $H \rightarrow \mu^{+} \mu^{-}$ the branching ratio is around $0.02 \%$.

Taking into account the previous numbers and the typical values of the product of the acceptance times efficiency of $30-40 \%$ for the final states without neutrinos $(\gamma \gamma, 4 \ell, \ell \ell \gamma, \mu \mu)$ and of 
$2-10 \%$ for the others, one can see that the number of Higgs boson candidates that are selected in the final sample used by ATLAS to study their properties is quite smaller than the half million initially produced by the LHC Run $1 p p$ collisions: "only" a couple of thousand candidates.

\section{The Higgs boson search: general approach and 2012 discovery}

In July 2012 both the ATLAS and CMS collaborations announced the discovery of a particle with mass near $125 \mathrm{GeV}$ and production rates consistent with those of a SM Higgs boson. Both experiments used approximately $10 \mathrm{fb}^{-1}$ of $p p$ collisions $\left(5 \mathrm{fb}^{-1}\right.$ at $7 \mathrm{TeV}$ and $5 \mathrm{fb}^{-1}$ at $\left.8 \mathrm{TeV}\right)$. The discovery was based on the combination of signals mainly in two channels, $H \rightarrow \gamma \gamma$ and $H \rightarrow Z Z^{*} \rightarrow 4 \ell$. In both final states, the signal was found and the production cross section was measured using this general approach:

- the final state of interest is identified, for instance by looking for events with two photons reconstructed in the detector;

- a selection is applied in order to suppress as much as possible the background while keeping a good signal efficiency. The selection exploits the differences between signal and background in terms of event topology and interactions of the particles produced with the detector, and is usually optimised on simulated signal and background samples. As an example, in the case of the di-photon final state, after the reconstruction a large fraction of the di-photon candidates is due to photon-jet and di-jet events in which one or two hadronic jets with a neutral meson $\left(\pi^{0}, \eta\right)$ carrying a large fraction of the jet momentum and disintegrating to two collimated photons are reconstructed as a single photon by the reconstruction algorithm. These "reducible" backgrounds are suppressed by requiring the reconstructed photon candidates to have energy deposits in the electromagnetic calorimeter that are consistent with those of an EM shower from a true, prompt photon. In addition the two photon candidates are required to be "isolated" from nearby hadronic activity, by requiring that the energy deposited in the hadronic calorimeter and the energy deposited in the EM calorimeter near a photon (typically in a cone of radius $\Delta R=0.4$ around the photon direction) is small.

- after the selection, the sample contains the signal and a remaining amount of background. A statistical procedure [33], based on a maximum-likelihood fit to one or more observables with different expected distributions for signal and background, is performed in order to determine the signal yield, $N_{S}$. In the fit, the signal distribution is usually estimated from simulated events, while that of the background(s) is obtained from a combination of simulation and data control samples. For the $H \rightarrow \gamma \gamma$ search, as well as for most of the searches presented here, the most obvious choice of observable to discriminate signal from background is the di-photon invariant mass, $m_{\gamma \gamma}$. Due to four-momentum conservation, the signal $m_{\gamma \gamma}$ distribution peaks at the true Higgs boson mass $m_{H}$, with a width dominated by the experimental photon energy resolution. For the background, which after the selection is dominated by non-resonant di-photon production, plus some residual contamination of photon-jet and di-jet events, the $m_{\gamma \gamma}$ distribution is smoothly decreasing, and its parameters can be determined directly from the data by looking at regions of $m_{\gamma \gamma}$ in which no signal is expected. 
- finally, the number of signal events that is measured is compared to the expected statistical fluctuations of the data in the background-only hypothesis, and discovery is conventionally claimed if the probability (" $p$-value") that the background fluctuates to yield a fake signal yield as large as $N_{S}$ is $3 \times 10^{-7}$, which corresponds to the area in the tails of a Gaussian distribution beyond $5 \sigma$. This probability is usually denoted as $p_{0}$, or $p$-value of the background. The signal production cross section times branching ratio is measured dividing the measured yield by the integrated luminosity of the data $L_{\mathrm{int}}$, and the signal acceptance and detection efficiency $A \times \varepsilon, \sigma \times B R=N_{S} /\left(A \times \varepsilon \times L_{\text {int }}\right)$. The results are often quoted in terms of the signal strength $\mu$, which is defined as the ratio between the measured signal yield and the SM prediction. In absence of a significant excess, upper limits are set on the production cross section at $95 \%$ confidence level $(C L)$ using a modified frequentist (CLs) method [34], by identifying the value of the cross section for which the $C L_{s}$ is equal to 0.05 .

The number of events (or the signal strength $\mu$ ) is obtained through the maximisation of the profile likelihood ratio $\Lambda(\alpha)=\frac{L(\text { data } \mid \alpha, \hat{\theta})}{L(\text { data } \mid \hat{\alpha}, \hat{\theta})}$, where $L$ is the likelihood function, $\alpha$ is the "parameter of interest" $\left(N_{S}\right.$ or $\left.\mu\right)$ and $\theta$ is the set of nuisance parameters, i.e. all other parameters of the likelihood, including background and signal model parameters and systematic uncertainties. Given the observed data, the likelihood function is maximised for $\alpha=\hat{\alpha}$ and $\theta=\hat{\theta}$; for a fixed value of alpha, the likelihood function is maximised for $\theta=\hat{\hat{\theta}}(\alpha)$. Maximising the likelihood (either inconditionally or for a fixed value of $\alpha$ ) with respect to its nuisance parameters is also called "profiling" and the nuisance parameters are said to be "profiled".

To increase the sensitivity to the Higgs boson signal, several decay channels are investigated and combined together, so that the probability of the background to fluctuate simultaneously in the different reconstructed final states to give fake signals is smaller. An additional strategy to increase the sensitivity to the signal is to use more than one discriminating variable in the final fit, in order to obtain a better separation between signal and background, either through a multi-dimensional fit to many observables or through a one-dimensional fit to the response of a multivariate algorithm combining several input observables in a single output variable.

Clearly, the probability that the background fluctuations can mimic a signal of a certain size depends on the signal and background cross sections and efficiencies, but also on the resolution for signal events on the discriminating variable $x$ used in the fit. This is due to the fact that background fluctuations can only mimic a signal if they take place in regions of $x$ where the signal is expected to be found. For this reason the decays to $\gamma \gamma$ and $Z Z^{*} \rightarrow 4 \ell$ final states, with an invariant mass resolution at the 1-2\% level, are the ones that provide the best sensitivity compared to other final states (like $\tau \tau, W W^{*}, b \bar{b}$ ) with higher production cross sections but larger backgrounds and worse (10-15\%) invariant mass resolution.

Figure 2 shows the invariant mass distribution of the di-photon and four-lepton selected candidates that lead to the Higgs boson discovery in July 2012. An excess with respect to the background near $125 \mathrm{GeV}$ is visible in both final states. The $p_{0}$ as a function of $m_{H}$ is shown in Fig. 3, after combining the results in these two channels with those of a search for $H \rightarrow W W^{*} \rightarrow e v \mu \nu$ with $8 \mathrm{TeV}$ data and with previously published results of searches for $H \rightarrow Z Z^{*}, W W^{*}, b \bar{b}$ and $\tau^{+} \tau^{-}$in $7 \mathrm{TeV}$ data. The maximum deviation from the background-only hypothesis takes place for $m_{H}=126 \mathrm{GeV}$ and has a significance of $5.9 \sigma$. 

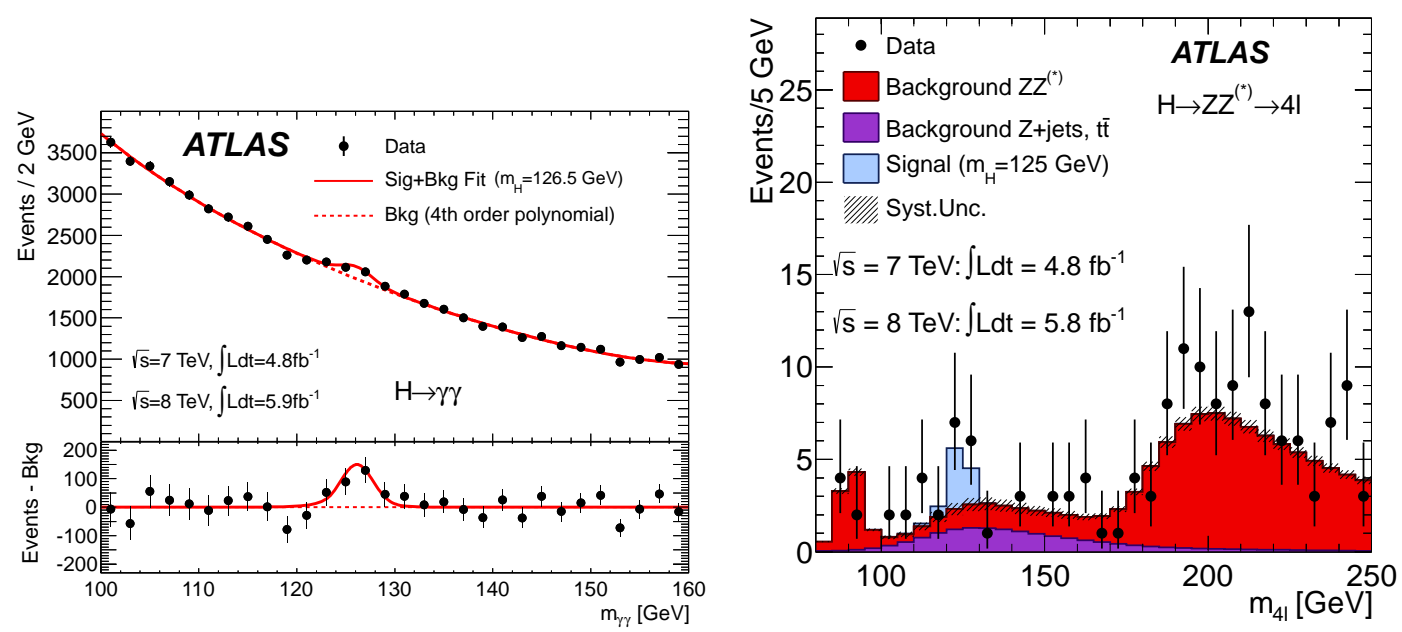

Figure 2: The distribution of the di-photon (left) and four-lepton (right) invariant masses for the selected candidates for the combination of the $\sqrt{s}=7 \mathrm{TeV}$ and $8 \mathrm{TeV}$ data analysed in July 2012. In the left plot the result of a fit to the data of the sum of a signal component fixed to $m_{H}=126.5 \mathrm{GeV}$ and a background component described by a fourth-order Bernstein polynomial is superimposed. In the right plot the data is compared to the expectation from the background plus a SM Higgs with $m_{H}=125 \mathrm{GeV}$. Taken from Ref. [14].

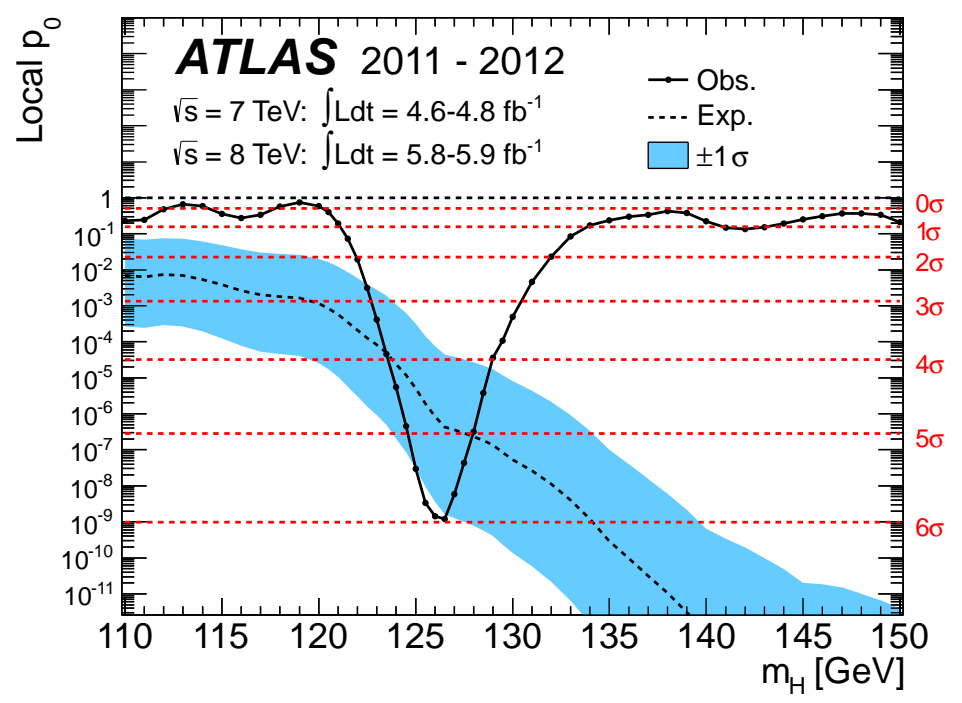

Figure 3: The observed (solid) local $p_{0}$ as a function of $m_{H}$ in the $110-150 \mathrm{GeV}$ range, for the combination of the $\sqrt{s}=7 \mathrm{TeV}$ and $8 \mathrm{TeV}$ data analysed in July 2012 The dashed curve shows the expected local $p_{0}$ under the hypothesis of a SM Higgs boson signal at that mass with its $\pm 1 \sigma$ band. The horizontal dashed lines indicate the $p$-values corresponding to significances of $1 \sigma$ to $6 \sigma$. Taken from Ref. [14]. 


\section{Higgs boson characterisation with the full ATLAS data from LHC Run1}

After the discovery of this new resonance, ATLAS has collected three times more data at $8 \mathrm{TeV}$ before the LHC stopped colliding protons and was shut down for two years (after a few weeks of heavy-ion collisions). An extensive program of characterisation of the newly discovered boson, based on this larger dataset, was undertaken and led to many important measurements of the properties of this particle, showing a good agreement with the expectations for a SM Higgs boson. In this section I will review such measurements.

\subsection{Higgs boson mass}

As already said, the SM does not predict the Higgs boson mass, even though constraints on it can be deduced from theoretical arguments like that from the unitarity of $W W$ scattering mentioned in the introduction. However, a precise measurement of the Higgs boson mass is crucial for any further Higgs boson study, for at least a couple of reasons. The first one is that in the SM the Higgs boson sector is determined by only two parameters, the vacuum expectation value of the Higgs field $v$, which is known, and $m_{H}$, so that once the Higgs boson mass is measured, all the partial widths, production cross sections and decay branching ratios can be calculated. The second one is that in the SM quantum corrections to many observables in the electroweak sector depend on the masses of the top quark $\left(m_{t}\right)$, of the $W$ boson $\left(m_{W}\right)$ as well as on $m_{H}$, so that a consistency test of the Standard Model can be performed by comparing direct $m_{t}$ and $m_{W}$ measurements to the indirect constraints obtained from fits to SM observables after fixing $m_{H}$, as shown in Fig. 4.

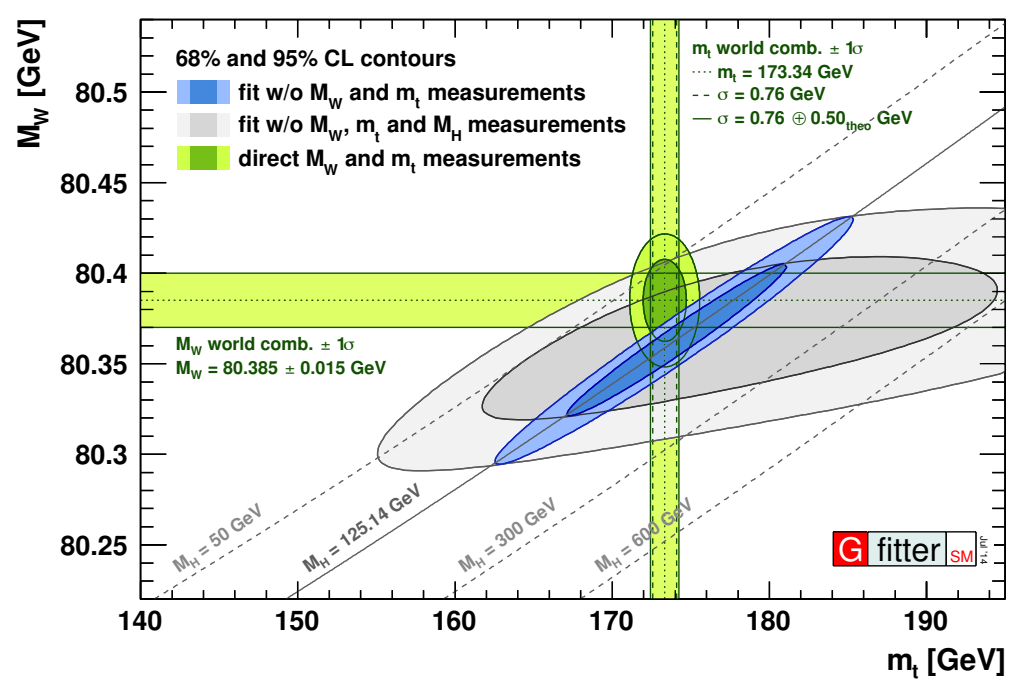

Figure 4: Contours of $68 \%$ and $95 \%$ confidence level obtained from scans of fits with fixed variable pairs $m_{W}$ vs $m_{t}$. The narrower blue and larger grey allowed regions are the results of the fit including and excluding the $m_{H}$ measurements, respectively. The horizontal bands indicate the $1 \sigma$ regions of the $m_{W}$ and $m_{t}$ measurements (world averages). Taken from Ref. [35]

Using the channels with largest significance and best invariant mass resolution, ATLAS has measured the mass $m_{H}$ by looking at the position of the di-photon and four-lepton invariant mass 
peaks [36]. For these results the latest energy-scale calibrations of photons, electrons and muons, based on larged samples of $Z$ and $J / \psi$ decays to di-leptons and radiative $Z \rightarrow \ell \ell \gamma$ decays, have been used. The mass is obtained through a fit to the invariant mass distributions in order to maximise the profile likelihood ratio $\Lambda\left(m_{H}\right)$. In the fit the signal strengths for the di-photon and the fourlepton channels are not constrained to the SM expectation $(\mu=1)$ nor, in the simultaneous fit to the combined dataset, to be identical to each other.

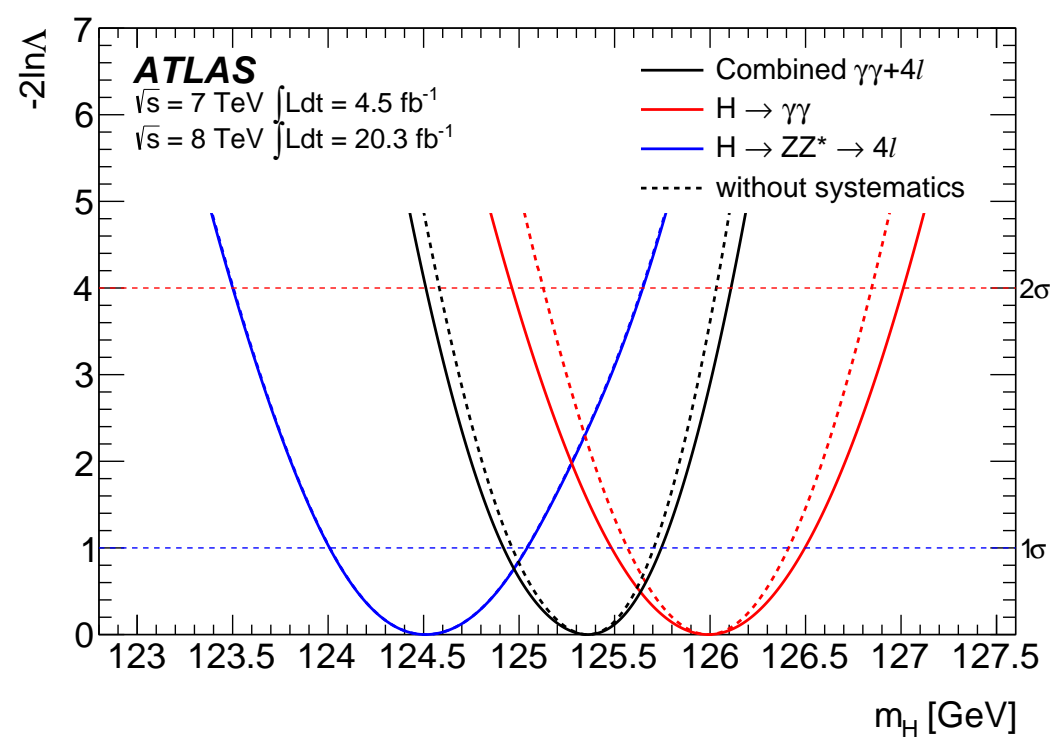

Figure 5: Value of $-2 \ln \Lambda$ as a function of $m_{H}$ for the individual $H \rightarrow \gamma \gamma$ and $H \rightarrow Z Z^{*} \rightarrow 4 \ell$ channels and their combination. The dashed lines show the statistical component of the mass measurements. The solid lines include the systematic uncertainties. Taken from Ref. [36].

The profile likelihood ratio as a function of the Higgs boson mass is shown in Fig. 5. The three functions obtained from the di-photon final state, the four-lepton final state and from the combination of the two datasets are shown. The mass is measured to be $m_{H}=125.36 \pm 0.37 \pm 0.18 \mathrm{GeV}^{3}$ The measurement is currently limited by the statistical uncertainty; the dominant sources of systematic uncertainties are the contributions to the electron and photon energy scale uncertainties from:

- the uncertainty on the amount of material in front of the calorimeter $(\approx 70 \mathrm{MeV})$;

- the uncertainty on the non-linearity of the energy measurement at the cell level, mostly from the relative calibration of the different gains used in the calorimeter readout $(\approx 70 \mathrm{MeV})$;

- the uncertainty on the relative calibration of the different calorimeter layers $(\approx 50 \mathrm{MeV})$;

- the uncertainty in the modeling of the lateral electromagnetic shower distribution in the simulation $(\approx 50 \mathrm{MeV})$.

\footnotetext{
${ }^{3}$ if not stated otherwise the first uncertainty is statistical and the second is the systematic one
} 
The level of consistency between the mass measurements in the two channels is estimated by allowing the mass to be different in the two channels $\left(m_{H}^{4 \ell}\right.$ and $m_{H}^{\gamma \gamma}$ ), rewriting the profile likelihood in terms of $m_{H}^{4 \ell}$ and of $\Delta m_{H}=m_{H}^{\gamma \gamma} m_{H}^{4 \ell}$, and profiling $m_{H}^{4 \ell}$ in the fit. The measured value of the mass difference in the two channels is $\Delta m_{H}=1.47 \pm 0.67 \pm 0.28 \mathrm{GeV}$, indicating a $2.0 \sigma$ compatibility between the two channels.

Recently these results were combined [37] with those obtained in a similar way by the CMS collaboration. In the fit the signal strengths of each channel are assumed to be the same between the two experiments. ${ }^{4}$. The combined measured mass of the Higgs boson is $m_{H}=125.09 \pm 0.21 \pm$ $0.11 \mathrm{GeV}$, with a remarkably small relative uncertainty, at the level of two per mil (still statistically limited). The mutual compatibility of the $m_{H}$ results from the four individual channels is tested using a profiled likelihood ratio with four masses in the numerator and a common mass in the denominator. The resulting compatibility, defined as the $p$-value of the fit, is $10 \%$.

\subsection{Higgs boson production and decay rate strengths}

The Higgs boson production and decay rate strengths are defined as the ratios between the measured values and the SM expectations of the production cross sections $\sigma_{i}$ (for a particular process $i$ ) and of the branching ratios $B R_{f}$ for the decay to various final states $f$ :

$$
\mu_{i}=\frac{\sigma_{i}}{\sigma_{i}^{\mathrm{SM}}}, \mu_{f}=\frac{B R_{f}}{B R_{f}^{\mathrm{SM}}}
$$

Such quantities are measured by reconstructing Higgs boson decays to many final states and identifying signal events produced by different production modes by exploiting their different topologies, as described in Sec. 3. This is achieved by classifying the selected events in event categories $c$, based on quantities like the presence of an additional same-flavour, opposite-sign dilepton pair with invariant mass near $91 \mathrm{GeV}$ (mostly $Z H$ events) of the presence of two high- $p_{\mathrm{T}}$ jets with large invariant mass and pseudorapidity separation (mostly VBF events, with an important contamination from gluon fusion events with simultaneous radiation of hard jets). After subtracting the background, the number of events in data in each category $c$ is given by:

$$
n_{s}^{c}=\sum_{i} \sum_{f} \mu_{i} \sigma_{i}^{\mathrm{SM}} \times \mu_{f} B R_{f}^{\mathrm{SM}} \times A_{i f}^{c} \times \varepsilon_{i f}^{c} \times L_{\mathrm{int}}^{c}
$$

where $A_{i f}^{c}$ is the acceptance for events of the type $i \rightarrow H \rightarrow f$ classified as belonging to category $c, \varepsilon_{i f}^{c}$ is the detection efficiency for such events that are in the detector acceptance, and $L_{\text {int }}^{c}$ is the integrated luminosity for the given category. The acceptances $A_{i f}^{c}$ are obtained, together with the $\mathrm{SM}$ cross sections and branching ratios $\sigma_{i}^{\mathrm{SM}}$ and $B R_{f}^{\mathrm{SM}}$, from theoretical calculations, assuming $m_{H}=125.36 \mathrm{GeV}$; the efficiencies $\varepsilon_{i f}^{c}$ are estimated from simulations of the detector response to signal events; and the integrated luminosity and the signal yields are measured experimentally. A maximisation of the profile likelihood ratio $\Lambda\left(\left\{\mu_{i}, \mu_{f}\right\}\right)$ then leads to an estimate of the signal strengths.

If the discovered particle is the SM Higgs boson all measured values of $\mu_{i}$ and $\mu_{f}$ should be equal to one. Viceversa, if all measured values of the strength factors are equal to one, then the

\footnotetext{
${ }^{4}$ in the di-photon channel, since CMS classifies the events in categories that are sensitive to different production mechanisms, two separate signal strengths $\mu_{g g F+t t H}^{\gamma \gamma}$ and $\mu_{V B F+V H}^{\gamma \gamma}$ are used
} 
discovered particle is most likely the SM Higgs boson or has properties very similar to it, since otherwise the product $A_{i f}^{c} \times \varepsilon_{i f}^{c}$ would be different from the one estimated using theoretical calculations and simulation based on the SM Higgs boson kinematics for at least one or few categories, unless subtle cancellations of SM deviations in the acceptance and the efficiency occur.

The measurement of the signal production and decay rate strengths is based on the analyses of several final states predicted by the SM. An overview of the analyses used as inputs is given in Table 1. The combination of such analyses is described in Ref. [38].

Table 1: Overview of the individual analyses that are included in the combination described in Sec. 5.2. The checkmark indicates whether the analysis classifies the events in categories that are sensitive to a particular Higgs boson production process.

\begin{tabular}{lcccccccc}
\hline \hline Decay mode & \multicolumn{6}{c}{ Sensitivity to production modes } & \multicolumn{2}{c}{ Observed significance } \\
& ggF & VBF & $V H$ & ttH & Expected significance & Ref. \\
& or 95\% CL limit & CL limit & \\
\hline$\gamma \gamma$ & $\checkmark$ & $\checkmark$ & $\checkmark$ & $\checkmark$ & $5.2 \sigma$ & $4.6 \sigma$ & {$[39,40]$} \\
$Z Z^{*} \rightarrow 4 \ell$ & $\checkmark$ & $\checkmark$ & $\checkmark$ & $\checkmark$ & $8.1 \sigma$ & $6.2 \sigma$ & {$[41,42]$} \\
$W W^{*}$ & $\checkmark$ & $\checkmark$ & $\checkmark$ & $\checkmark$ & $6.5 \sigma$ & $5.9 \sigma$ & {$[42,43,44]$} \\
$\tau^{+} \tau^{-}$ & $\checkmark$ & $\checkmark$ & & & $4.5 \sigma$ & $3.4 \sigma$ & {$[42,45]$} \\
$b \bar{b}$ & & & $\checkmark$ & $\checkmark$ & $1.4 \sigma$ & $2.6 \sigma$ & {$[46,47]$} \\
$Z \gamma$ & $\checkmark$ & $\checkmark$ & & & $\mu<11$ & $\mu<9$ & {$[48]$} \\
$\mu^{+} \mu^{-}$ & $\checkmark$ & $\checkmark$ & & & $\mu<7.0$ & $\mu<7.2$ & {$[49]$} \\
\hline \hline
\end{tabular}

Among the final states being selected, the one with the best $S / B$ is $Z Z^{*} \rightarrow 4 \ell$, with an $S / B \approx$ 1.5; other final states have typical $S / B$ of a few $\%$ after the full selection, with the exception of the rare decays $H \rightarrow Z \gamma$ and $H \rightarrow \mu \mu$, for which $S / B$ is at the level of a few per mil, due to nonresonant $Z+\gamma$ and to $Z \rightarrow \mu \mu$ events. The invariant mass resolution is at the $1-2 \%$ level for $\gamma \gamma$, $Z Z^{*} \rightarrow 4 \ell, Z \gamma \rightarrow \ell \ell \gamma$ and $\mu \mu$, and about ten times larger for $H \rightarrow W W^{*}, \tau \tau$ and $b \bar{b}$. As can be seen from the table, the bosonic Higgs boson decays $\left(\gamma \gamma, Z Z^{*}, W W^{*}\right)$ are clearly observed, except for the $Z \gamma$ final state. Among the fermionic decays, a strong evidence is found for the leptonic decay $H \rightarrow \tau \tau$, while only a weak hint of $H \rightarrow b \bar{b}$ decays is observed and there is no evidence of $H \rightarrow \mu^{+} \mu^{-}$decays.

Assuming a common multiplier $\mu$ to all production cross sections and decay branching ratios, the observed event yields in each category lead to a global signal-strength value of

$$
\mu=1.18 \pm 0.10(\text { stat }) \pm 0.07(\text { syst })_{-0.07}^{+0.08}(\text { theo })=1.18_{-0.14}^{+0.15}
$$

where the labels stat., syst. and theo. refer to statistical, systematic, and signal theoretical uncertainties, respectively. The signal theoretical uncertainty includes contributions from uncertainties in SM cross sections and branching ratios as well as in the modeling of the production and decays of the Higgs boson. The largest source of experimental systematic uncertainty is from background estimates in the analyses of individual channels. This result is consistent with the SM expectation of $\mu=1$, with a $p$-value of $18 \%$.

Figure 6 shows the signal strength factors for different production modes and different decay modes. The former are obtained assuming SM values for the ratios of the branching ratios 

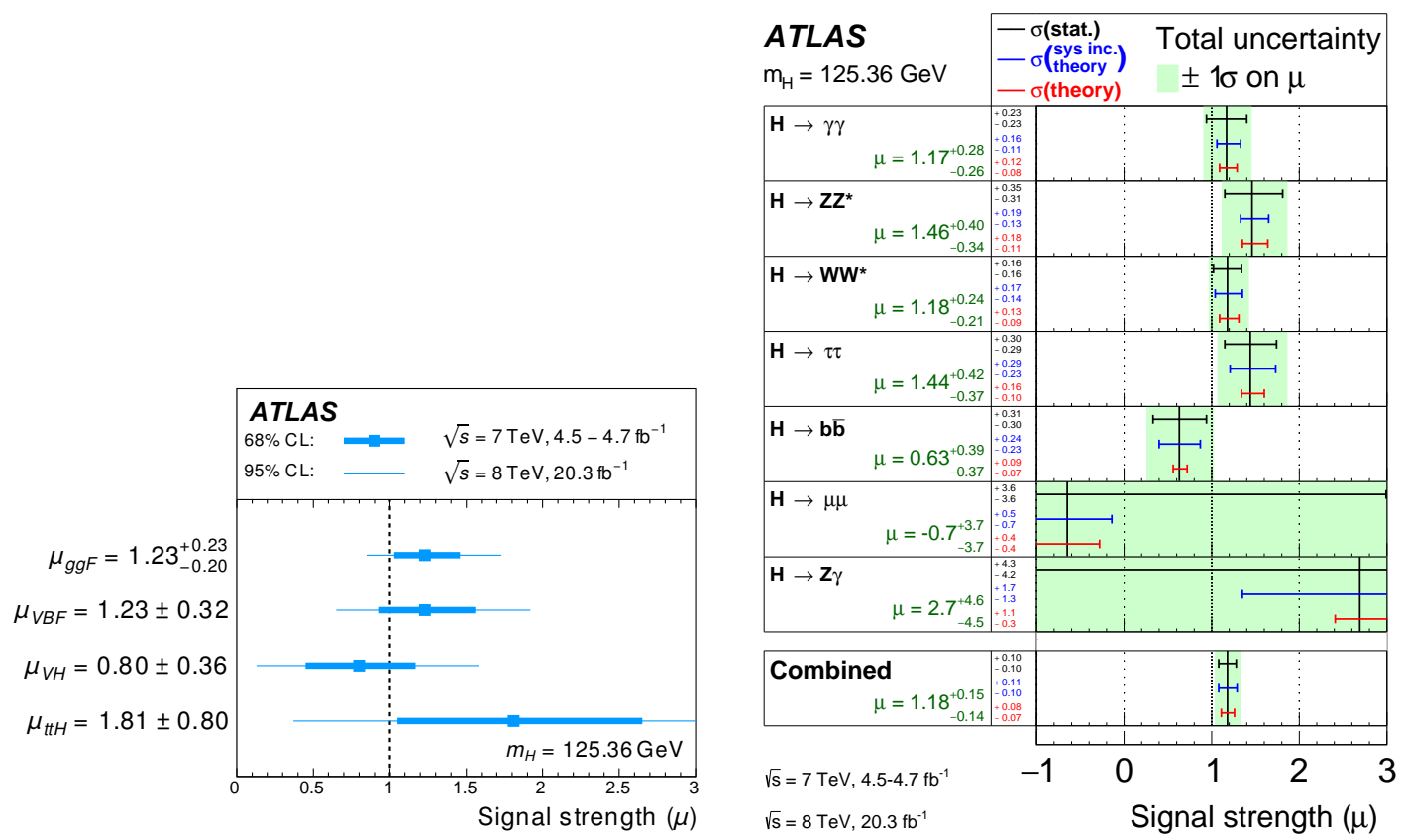

Figure 6: Left: best-fit signal-strength values of different production modes, assuming SM Higgs boson decay branching ratios for $m_{H}=125.36 \mathrm{GeV}$. The inner and outer error bars correspond to 68\% CL and 95\% $\mathrm{CL}$ intervals. Total uncertainties combining statistical, experimental and theoretical systematic uncertainties are shown. Right: the observed signal strengths and uncertainties for different Higgs boson decay channels and their combination for $m_{H}=125.36 \mathrm{GeV}$, assuming SM values for the cross-section ratios of different production processes. Taken from Ref. [38].

of different Higgs boson decays and with some simplifying hypothesis on the cross sections of the rarer production processes $\left(\mu_{b b H}=\mu_{g g F}, \mu_{t H}=\mu_{t t H}, \mu_{W H}=\mu_{Z H}\right)$. The latter are obtained assuming SM values for the cross-section ratios of different production processes. All strengths are consistent with the SM expectation of one. All individual measurements of the signal-strength parameters are consistent and compatible with the combined value, with a $p$-value of $76 \%$. Among the production modes, ggF is clearly observed with a $>5 \sigma$ significance, and a $>4 \sigma$ evidence of VBF production is found; there is no evidence yet of $V H$ (although evidence for it is provided by the results of the CDF and D0 experiments) and of $t t H$. Combining the results from various analyses with sensitivity to $t t H$ production, its strength if found to be larger than one by slightly more than one standard deviation; the observed and expected upper limits on it are $\mu_{t t H}<3.2$ and 1.4 , respectively.

Similar measurements have been performed by the CMS collaboration [50] on a dataset of similar size. Combining the ATLAS and CMS results together [51] yields a global signal strength $\mu=1.09_{-0.10}^{+0.11}$, whose uncertainty is dominated equally by statistical and theory systematic uncertainties. All production and decay rate strengths are in agreement with the SM. The combined significance of VBF production and of $H \rightarrow \tau \tau$ decays exceed $5 \sigma$; the latter provides the first direct observation of couplings of the discovered particle to fermions. Intriguingly, also CMS finds hints of an excess of $t t H$ production with respect to the SM expectation; the combined best-fit value of 
$\mu_{t t H}$ is $2.3_{-0.6}^{+0.7}$, leading to a $\approx 2 \sigma$ excess with respect to the SM prediction and to an evidence of $t t H$ production at the $4.4 \sigma$ level.

\subsection{Higgs boson total and differential production cross sections}

The total Higgs boson production cross-sections for each production mode are obtained by multiplying the SM predictions by the measured production strengths $\mu_{i}$. The theoretical uncertainties on the absolute values of the SM Higgs boson production cross sections are removed from $\mu_{i}$. Summing the cross sections of the different production modes the total cross sections are determined to be:

$$
\begin{aligned}
& \sigma_{H}(7 \mathrm{TeV})=22.1_{-6.0}^{+7.4} \mathrm{pb}=22.1_{-5.3}^{+6.7}(\text { stat. })_{-2.3}^{+2.7}(\text { syst. })_{-1.4}^{+1.9}(\text { theo. }) \mathrm{pb} \\
& \left.\left.\sigma_{H}(8 \mathrm{TeV})=27.7 \pm 3.7 \mathrm{pb}=27.7 \pm 3.0(\text { stat. })_{-1.7}^{+2.0} \text { (syst. }\right)_{-0.9}^{+1.2} \text { (theo. }\right) \mathrm{pb}
\end{aligned}
$$

They are larger by about one standard deviation than the theoretical predictions of $17.4 \pm 1.6 \mathrm{pb}$ at $7 \mathrm{TeV}$ and $22.3 \pm 2.0 \mathrm{pb}$ at $8 \mathrm{TeV}$. The uncertainties on the measurement are dominated by the gluon-fusion contribution.

Measurements of the differential Higgs boson production cross sections have been performed with the $8 \mathrm{TeV}$ data using the events selected in the final states with the largest significance, $H \rightarrow \gamma \gamma$ $H \rightarrow Z Z \rightarrow 4 \ell$ [52]. The measured cross sections probe the properties of the Higgs boson and can be directly compared to the theoretical modeling of different Higgs boson production mechanisms. Differential cross sections are measured as a function of several observables that are sensitive to various theoretical effects: perturbative QCD modelling in ggF production (Higgs boson transverse momentum $p_{\mathrm{T}}^{H}$ and rapidity $y^{H}$ ), modeling of partonic radiation in ggF as well as the overall rate and modeling of jets in VBF, $V H$ and $t t H$ events (number of jets $N_{\text {jets }}$ in the event and $p_{\mathrm{T}}$ of the leading jet, $\left.p_{\mathrm{T}}^{j 1}\right)$. The differential cross sections are determined from the background-subtracted events yields taking into account detector efficiencies, fiducial acceptances and (SM) branching ratios to the two final states under study. The selected events are not classified in the productionmode sensitive categories used for the measurement of the signal strengths; rather, differential yields as a function of the observable under study are extracted, either from fits to the diphoton mass spectra for the $H \rightarrow \gamma \gamma$ channel, or from the background subtracted data yield in a $m_{4 \ell}$ mass window of 118 to $129 \mathrm{GeV}$ for the $H \rightarrow Z Z^{*} \rightarrow 4 \ell$ channel. The measurements in the two channels are found to be in good agreement with each other, with $p$-values from compatibility tests in the range $56-99 \%$, and then combined. Statistical uncertainties dominate all resulting distributions, ranging from $23 \%$ to $75 \%$. The measurements are compared to state-of-the-art predictions, as shown in Fig. 7. The shapes of the distributions measured in data are found in good agreement with the predictions, with $p$-values between $8 \%$ and $88 \%$, while the overall normalisation is larger in data by approximately $50 \%$ ( 2 standard deviations) of the prediction: the total production cross section is determined to be $\sigma(p p \rightarrow H)=33.0 \pm 5.3 \pm 1.6 \mathrm{pb}$.

The differential cross sections measured with the $H \rightarrow \gamma \gamma$ final state are also used to set constraints on beyond-SM (BSM) Higgs boson interactions within an effective field theory framework [53]. The cross-sections are compared to the predictions of an effective lagrangian that includes new $C P$-even and $C P$-odd interactions between the Higgs boson and photons, gluons, $\mathrm{W}$-bosons and Z-bosons. Such interactions are forbidden by the SM and can lead to changes in 

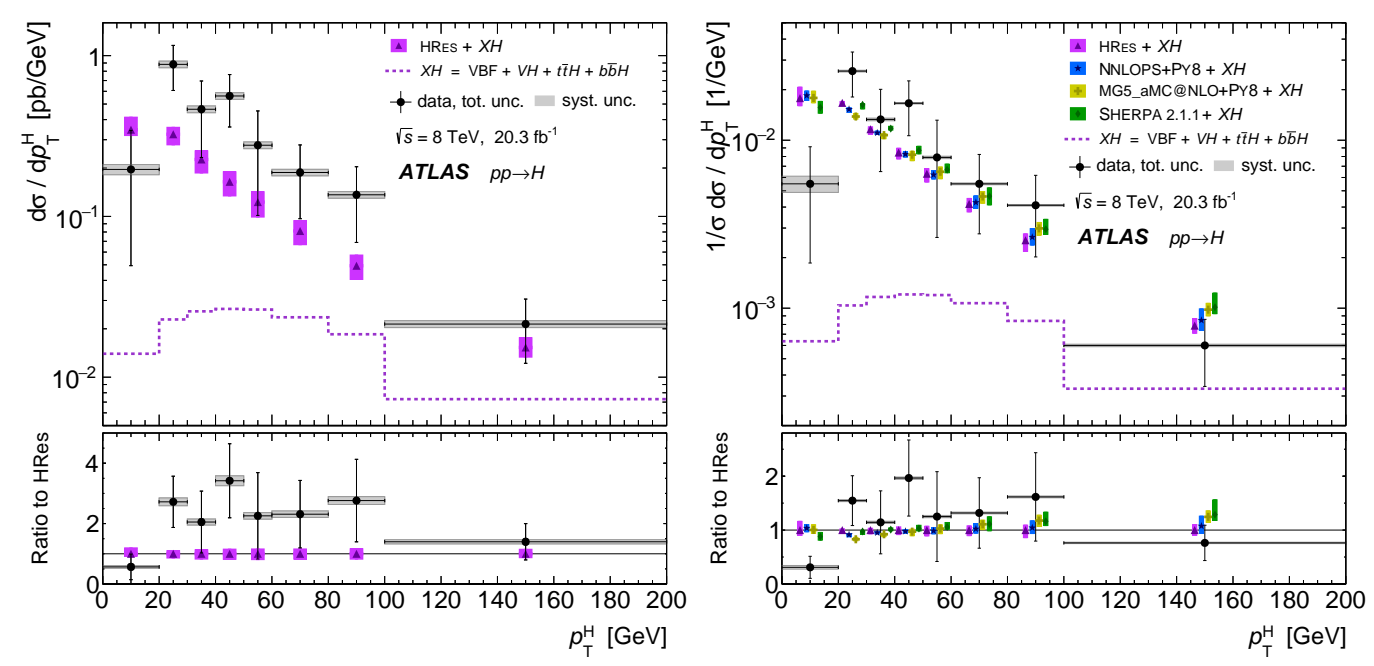

Figure 7: Differential cross sections (left) and normalized cross-section shapes (right) for inclusive Higgs boson production measured by combining the $H \rightarrow \gamma \gamma$ and $H \rightarrow Z Z^{*} \rightarrow 4 \ell$ channels. The measured variable is the Higgs boson transverse momentum. Various theoretical predictions are presented, using the same bin widths as the measurement: a theoretical NNLO+NNLL calculation (HRES) and various Monte Carlo event generators (POWHEG NNLOPS, MadGraph5_aMC@NLO, SHERPA 2.1.1). Taken from Ref. [52].

the kinematic properties of the Higgs boson and of the associated jet spectra. The leading-order predictions obtained from Madgraph5, interfaced to PYTHIA6 to model hadronisation and underlying event, are reweighted to account for higher-order QCD and electroweak corrections to the SM process, assuming that these corrections factorise from the new physics effects. A simultaneous fit to five differential cross sections leads to constraints on the parameters of the effective lagrangian. All the coefficients of the terms of the lagrangian leading to new physics effects are found to be consistent with zero and limits on their magnitude are set at levels between 0.2 and $2 \times 10^{-4}$.

\subsection{Couplings of the Higgs bosons to the SM particles}

The coupling strengths of the Higgs boson to SM vector bosons and fermions in different benchmark models are determined from a combined analysis [38] of the measurements presented in Sec. 5.2. The analysis assumes that the signals observed in the different channels originate from a single narrow resonance with a mass near $125.36 \mathrm{GeV}$, that the production and decay kinematics of the discovered particle are compatible with the SM expectation, and that the tensor structure of its couplings is the same as in the SM (i.e. a pure CP-even state).

In the zero-width approximation, the cross section $\sigma(i \rightarrow H \rightarrow f)$ for on-shell measurements is written as

$$
\sigma(i \rightarrow H \rightarrow f)=\frac{\sigma_{i}\left(\left\{\kappa_{j}\right\}\right) \Gamma_{f}\left(\left\{\kappa_{j}\right\}\right)}{\Gamma_{H}\left(\left\{\kappa_{j}\right\}\right)}
$$

where $\sigma_{i}$ is the Higgs boson production cross section through the initial state $i, \Gamma_{f}$ its partial decay width into the final state $f, \Gamma_{H}$ the Higgs boson total width, and each $\kappa_{j}$ is the scale factor of the Higgs boson coupling strength to the particle $j\left(\kappa_{j}=1\right.$ in the SM). The leading-order expressions for the cross sections and partial widths are used to parametrise their dependence on the coupling- 
strength modifiers: for instance, the cross section $\sigma_{W H}$ is written as $\sigma_{W H}=\kappa_{W}^{2} \sigma_{W H}^{S M}$, and the partial width $\Gamma_{\tau \tau}$ is $\Gamma_{\tau \tau}=\kappa_{\tau}^{2} \Gamma_{\tau \tau}^{S M}$.

In some of the fits, effective scale factors $\kappa_{g}, \kappa_{\gamma}$ and $\kappa_{Z \gamma}$ are introduced to describe the processes $g g \rightarrow H, H \rightarrow \gamma \gamma$ and $H \rightarrow Z \gamma$, which are loop-induced in the SM. In other fits they are treated as a function of the coupling-strength scale factors to the particles that contribute to these SM loop processes: for instance, the di-photon partial width modifier is $k_{\gamma}^{2}=1.59 \kappa_{W}^{2}+0.07 \kappa_{t}^{2}-$ $0.66 \kappa_{W} \kappa_{t}$, taking into account the contributions from $W$ and top-mediated loop amplitudes and their interference (which, by the way, allows their relative sign to be probed).

The Higgs boson total width depends on invisible or undetected decays $\left(\Gamma_{H}=\frac{\kappa_{H}^{2}\left(\kappa_{j}\right)}{1-B R_{i, u}} \Gamma_{H}^{S M}\right)$; since such decays are not experimentally constrained, only ratios of coupling strengths can thus be measured by ATLAS without assumptions about $\Gamma_{H}$. To make measurements of absolute coupling strengths, some assumptions must be made. In some cases, the branching ratio $B R_{i, u}$. to invisible or undetected decays is assumed to be zero. In other cases, the coupling strength modifiers for vector bosons $k_{V}$ are assumed not to exceed one, as is the case in many BSM models and as necessary if the Higgs boson should solve the unitarity problem in vector boson scattering. A third alternative is to assume that the coupling strength scale factors for on-shell Higgs boson production are identical to those for off-shell Higgs boson production. Constraints on the off-shell couplings are set looking at $Z Z(\rightarrow 4 \ell, 2 \ell 2 v)$ and $W W(\rightarrow e v \mu v)$ events in the mass range above the $2 m_{Z}$ and $2 m_{W}$ thresholds [54]. The off-shell signal strength at an energy scale $\hat{s}$ is

$$
\mu_{\text {off-shell }}(\hat{s}) \equiv \frac{\sigma_{\text {off-shell }}^{g g \rightarrow H^{*} \rightarrow V V}(\hat{s})}{\sigma_{\text {off-shell }, \text { SM }}^{g g \rightarrow H^{*} \rightarrow V V}(\hat{s})}=\kappa_{g, \text { off-shell }}^{2}(\hat{s}) \kappa_{V, \text { off-shell }}^{2}(\hat{s}),
$$

where $\kappa_{g, \text { off-shell }}^{2}(\hat{s})$ and $\kappa_{V, \text { off-shell }}$ are the off-shell coupling scale factors associated with the $g g \rightarrow H^{*}$ production and the $H^{*} \rightarrow V V$ decay. Due to the statistically limited sensitivity of the analysis, the dependence of the off-shell signal strength and coupling scale factors on $\hat{s}$ is neglected. Interference effects with the $g g \rightarrow V V$ background are large, of negative size, and accounted for in the analysis. Other simplifying hypotheses are:

- the background predictions are nor modified by any new physics which modifies the off-shell couplings;

- there are nosizeable kinematic modifications to the off-shell signal nor new, sizeable signals in the search region of the analysis unrelated to an enhanced off-shell signal strength;

- the ratio between the $k$-factors for $g g \rightarrow V V$, which is unknown, and that of $g g \rightarrow H^{*} \rightarrow V V$, which is calculated, is in the range 0.5-2.0;

- to combine the results of the two channels, the ratio between the $Z Z$ and $W W$ cross sections is assumed to be as predicted in the SM for both the on- and off-shell processes.

Figure 8 shows the $Z Z$ invariant mass distributions in data used to constrain (with similar distributions from $W W$ decas) $\mu_{\text {off-shell }}$. A 95\% CL upper limit of 8.1 is set on $\mu_{\text {off-shell }}$; without systematic uncertainties, the limit of 6.5. The dominant systematic uncertainties originate from theoretical uncertainties on the interference between the $g g \rightarrow H^{*} \rightarrow V V$ and the $g g \rightarrow V V$ processes and on the $\mathrm{K}$-factor of the two processes. 

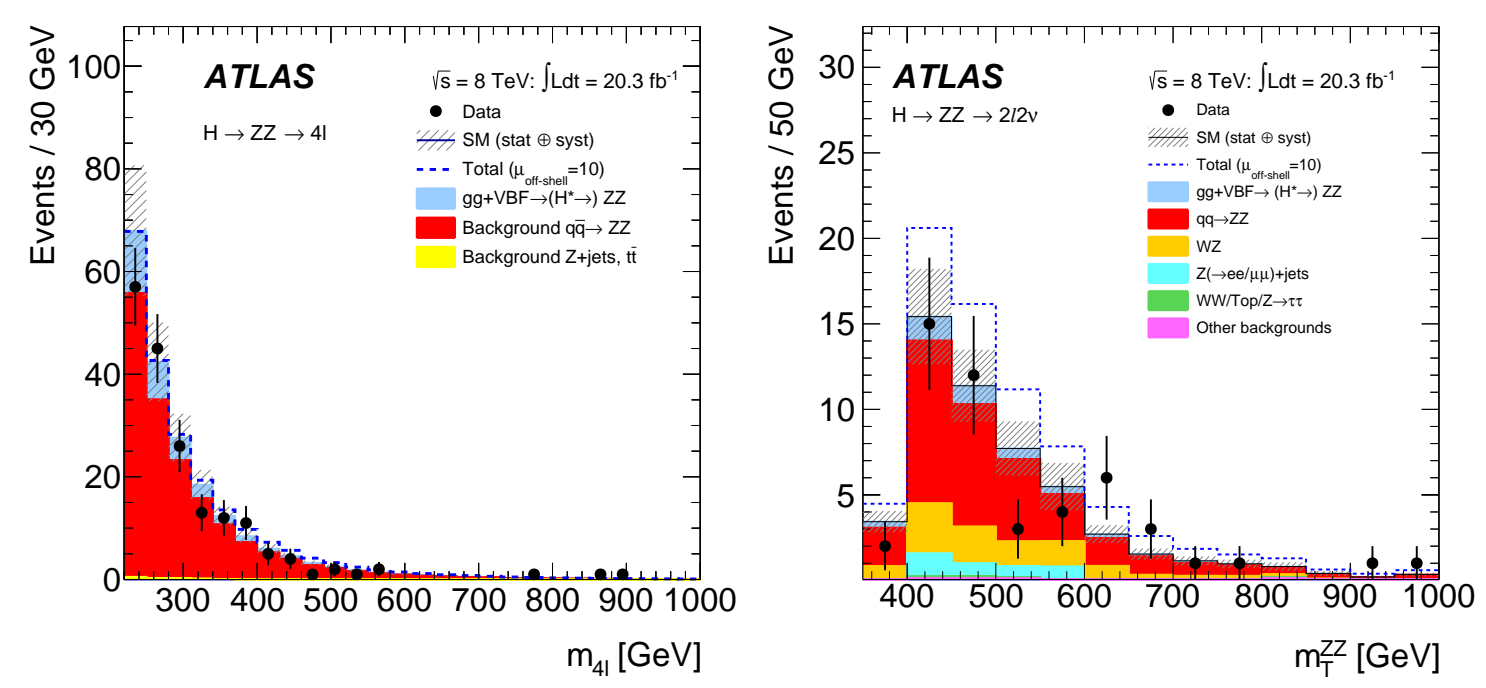

Figure 8: Observed distributions for the four-lepton invariant mass $m_{4 \ell}$ in the range $220 \mathrm{GeV}<m_{4 \ell}<1000$ $\mathrm{GeV}$ (left) and the $Z Z$ transverse mass $m_{\mathrm{T}}^{Z Z}$ in the range $380 \mathrm{GeV}<m_{\mathrm{T}}^{Z Z}<1000 \mathrm{GeV}$ combining the $2 e 2 v$ and $2 \mu 2 v$ channels (right), compared to the expected contributions from the SM including the Higgs boson (stack). The dashed line corresponds to the total expected event yield, including all backgrounds and the Higgs boson with off-shell $=10$. A relative $g g \rightarrow Z Z / g g \rightarrow H^{*} \rightarrow Z Z K$-factor of 1.0 is assumed. Taken from Ref. [54].

Many benchmark models are tested and the corresponding coupling strength modifiers are extracted, including:

- models with universal coupling-strength scale factors $\kappa_{F}$ for all fermions and $\kappa_{V}$ for all vector bosons (with no undetected or invisible Higgs boson decay, or assuming that loop processes have only SM content);

- models with universal coupling-strength scale factors $\kappa_{d}, \kappa_{u}$ and $\kappa_{V}$ for down-type fermions, up-type fermions and vector bosons, respectively (assuming that loop processes have only SM content);

- models with universal coupling-strength scale factors $\kappa_{l}, \kappa_{q}$ and $\kappa_{V}$ for leptons, quarks and vector bosons, respectively (assuming that loop processes have only SM content);

- models with SM couplings to SM particles but with BSM contributions in loop vertices (thus modifying $\left.\kappa_{g}, \kappa_{\gamma}, \kappa_{Z \gamma}\right)$ and possibly also to the total width;

- generic models with or without new particles in loops and in decay. Such models include also the case when the $S U(2)$ custodial symmetry of the SM is violated $\left(\kappa_{W} \neq \kappa_{Z}\right)$.

All tested configurations yield coupling strength scale factors (or ratios of coupling strength scale factors in case no assumption about the total width is applied) consistent with the SM; this is shown in Figure 9 for the case of generic models with a large number of coupling scale factors allowed to be different from one. The negative sign of $\kappa_{t}$ relative to $\kappa_{W}$ is disfavoured. 

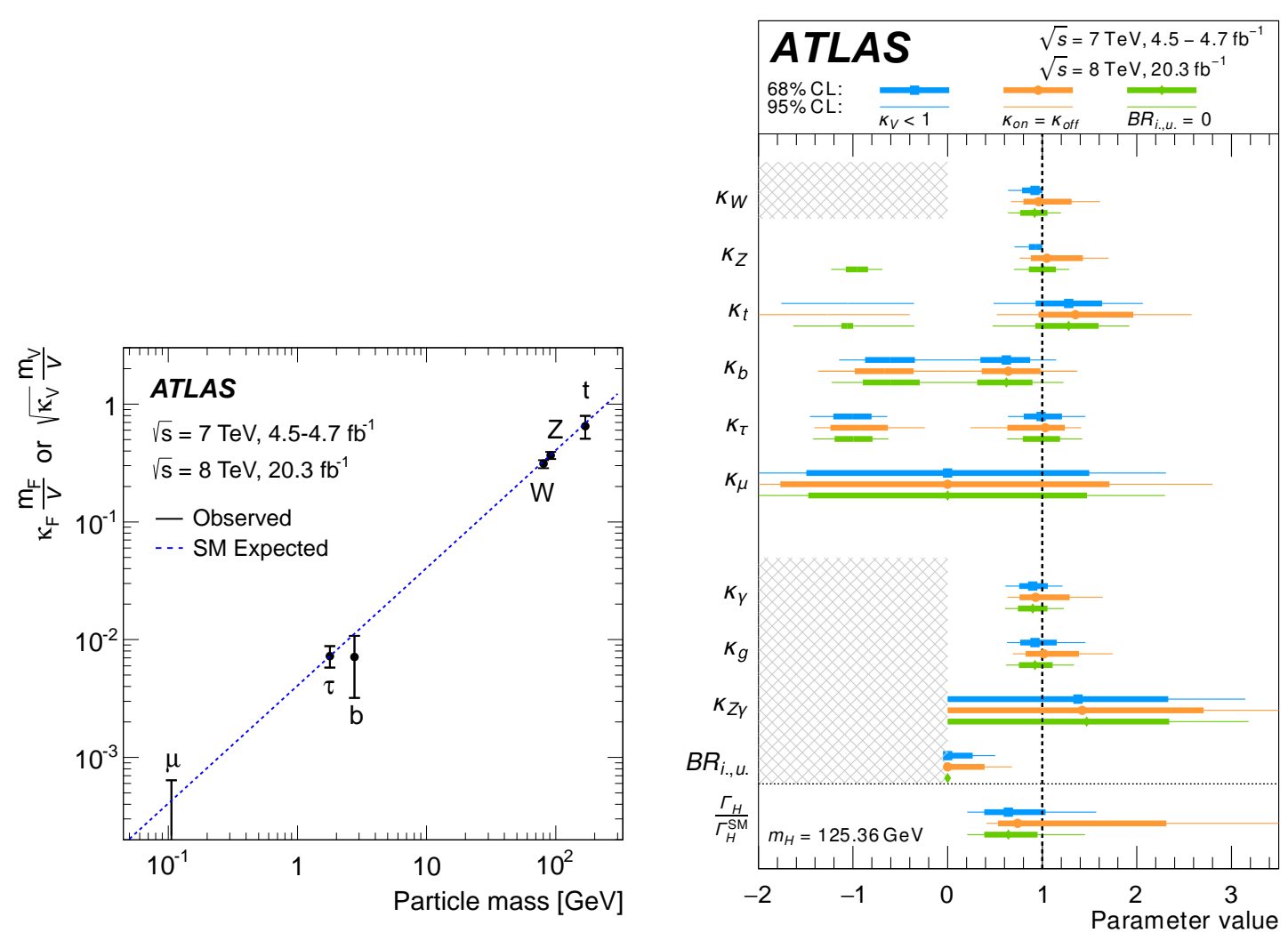

Figure 9: Left: measured reduced coupling-strength scale factors $\sqrt{\kappa_{V}, i} \frac{m_{V, i}}{v}$ for weak bosons and $\kappa_{F, i} \frac{m_{F, i}}{v}$ for fermions as a function of the particle mass, in generic models in which no new particles contribute to the loops and to the total width. The six coupling strength scale factors shown here are the only free parameters of the model. The dashed line indicates the predicted mass dependence for the SM Higgs boson Right: measured coupling-strength scale factors for generic models in which new particles can contribute to loops and to the total width. The estimated value of each parameter under either the constraint $\kappa_{V}<1, \kappa_{\text {on }}=\kappa_{\text {off }}$ or $B R_{i, u}$. $=0$ are shown with markers in the shape of a box, a circle, or a diamond, respectively. The hatched area indicates regions that are outside the defined parameter boundaries. The inner and outer bars correspond to $68 \% \mathrm{CL}$ and $95 \% \mathrm{CL}$ intervals. Taken from Ref. [38].

Combining the ATLAS results with similar ones from CMS [50, 51] leads to results that are still consistent with SM expectations, with reduced uncertainties. In particular, in the generic model with no new particles contributing to loops and decays, the uncertainties on the measured coupling scale factors are about $10 \%(W, Z), 14 \%(t, \tau)$, and $21 \%(b)$, respectively.

\subsection{Higgs boson width}

In the $\mathrm{SM}$ the total width of the Higgs boson for $m_{H}=125.36 \mathrm{GeV}$ is expected to be $4.12 \mathrm{MeV}$ and thus significantly smaller than the detector resolution even in the channels with the best resolution (of the order of 1.5-3 GeV), i.e. $H \rightarrow \gamma \gamma$ and $H \rightarrow Z Z^{*} \rightarrow 4 \ell$.

A direct limit on the decay width of the Higgs boson is set from the observed width of the invariant mass peak in these two channels, under the assumption that there is no interference with 
background processes. In the fit to the data, the signal probability density function entering the likelihood ratio is the convolution between the detector resolution function and a nonrelativistic Breit-Wigner with floating width $\Gamma_{H}$ [36]. Such studies yield upper limits on the Higgs boson width that are three orders of magnitude larger than the SM prediction: $5.0 \mathrm{GeV}$ and $2.6 \mathrm{GeV}$ in the di-photon and four-lepton channels, respectively, at 95\% CL.

An indirect limit on the decay width is obtained from an alternative approach, based the study of off-shell Higgs boson production described in the previous section, from the ratio between the off-shell and the on-shell signal strengths [54]. This approach exploits the fact that the off-shell signal strength is independent of the Higgs boson total width (see Eq. 5.7), while the on-shell signal strength depends on $\Gamma_{H}$ :

$$
\mu_{\text {on-shell }}(\hat{s}) \equiv \frac{\sigma_{\text {on }- \text { shell }}^{g g \rightarrow V V}(\hat{s})}{\sigma_{\text {on-shell,SM }}^{g g \rightarrow H}(\hat{s})}=\frac{\kappa_{g, \text { on-shell }}^{2}(\hat{s}) \kappa_{V, \text { on-shell }}^{2}(\hat{s})}{\Gamma_{H} / \Gamma_{H}^{S M}} .
$$

With the same simplifying hypotheses described for the extraction of the off-shell coupling strength scale factors, the $95 \% \mathrm{CL}$ upper limit on $\Gamma_{H}$ is $23 \mathrm{MeV}$.

\subsection{Higgs boson spin and parity}

The SM Higgs boson is a scalar $\left(J^{P C}=0^{++}\right)$. Experimentally the spin and parity of the particle discovered in July 2012 with mass near $125 \mathrm{GeV}$ can be determined from some kinematic distributions of signal events in $H \rightarrow \gamma \gamma, H \rightarrow Z Z^{*} \rightarrow 4 \ell$ and $H \rightarrow W W^{*} \rightarrow \ell v \ell v$ decays. In the di-photon channel, kinematic variables sensitive to the spin of the resonance are the di-photon transverse momentum and the production angle of the two photons. In the four-lepton channel, the distributions of the five production and decay angles depend on the spin and parity of the resonance. In the $W W^{*} \rightarrow \ell v \ell v$ channel, due to the spin correlations between the leptons arising from angular momentum conservation, several kinematic distributions (the di-lepton invariant mass, transverse momentum, azimuthal separation and the transverse mass of the leptons and of $E_{\mathrm{T}}^{\mathrm{miss}}$ ) are sentitive to the spin and parity of the resonance.

The pure $J=1$ hypothesis is excluded from the observation of the $H \rightarrow \gamma \gamma$ decay (LandauYang theorem) as well as from previous studies of $H \rightarrow Z Z^{*}$ and $H \rightarrow W W^{*}$ decays [55]. In the most recent study of the Higgs boson spin and parity by ATLAS [56], the SM hypothesis is compared to alternative spin- 0 models: a pseudoscalar boson $J^{P}=0$ and a BSM scalar boson $J^{P}=0_{h}^{+}$, which describes the interaction of the Higgs boson with the SM vector bosons with higher-dimension operators. Graviton-like tensor models with $J^{P}=2^{+}$with universal and nonuniversal couplings to quarks and gluons are also considered. In addition to the fixed spin and parity hypothesis tests, the possible presence of BSM terms in the Lagrangian describing the $H V V$ vertex of the spin- 0 resonance is also investigated. The HVV interaction is described in terms of an effective Lagrangian that contains the SM interaction and BSM CP-odd and CP-even terms. An effective field theory approach is adopted to describe the interaction between the resonance and the SM vector boson. After subtracting the contribution from background events, the distributions of the selected observables in the three bosonic decay channels are in good agreement with the $0^{+}$ hypothesis, disfavouring the alternative models considered at more than 99.9\% CL. 


\subsection{Beyond-SM Higgs boson decays}

All the measured properties of the resonance discovered in July 2012 so far are consistent with the SM expectations (width, spin, parity, couplings to all other SM particles). However, the level of precision of such measurements is sometimes quite limited (in particular, for the couplings to most elementary particles). Moreover, there are theoretical reasons for believing that the SM is not the ultimate theory of nature, but only its low-energy, approximate (though in excellent agreement with the data), version.

Many BSM theories predict a richer Higgs boson sector, with either more final that can be produced in the decay of the Higgs boson or with additional Higgs bosons. Studies of the Higgs boson sector are thus a portal to the investigation of such models. ATLAS has performed many searches of "exotic" (i.e. strongly suppressed or forbidden in the SM) decays of the Higgs boson discovered with mass near $125 \mathrm{GeV}$, including recent searches of:

- lepton flavour violating decays $(H \rightarrow \mu \tau)$ [57];

- decays to invisible final states [58, 59, 60, 61];

- decays to $J / \psi \gamma$ and $\Upsilon \gamma[62]$;

- decays to "dark" gauge bosons $Z_{D}\left(H \rightarrow Z Z_{D} \rightarrow 4 \ell\right)[63]$.

ATLAS has also performed searches of additional Higgs bosons, including recent searches of:

- a pseudoscalar $A$ boson decaying to $Z H(125)$ [64];

- a scalar boson decaying to $\mathrm{ZZ}(\rightarrow 4 \ell, 2 \ell 2 v, 2 \ell 2 q, 2 v 2 q)$ [65];

- a scalar boson decaying to di-photons [66];

- heavy charged Higgs bosons decaying to $W Z$ [67] and $\tau \nu$ [68].

No deviation from the SM found in any of such searches. In the $H \rightarrow \tau \mu$ decay channel, in which CMS observed a $2.5 \sigma$ excess, in the $8 \mathrm{TeV}$ data, ATLAS finds an excess with only a $1.3 \sigma$ significance, and sets an upper limit on the branching ratio of $1.85 \%$ at $95 \%$ CL. Combining the results of the searches of invisible decays of Higgs bosons produced either in association with vector bosons decaying to lepton or quark pairs or in VBF, an upper limit of $23 \%$ on the invisible branching ratio of the Higgs boson is set at $95 \%$ CL. Limits on the production cross sections of extra Higgs bosons are set at the level of few fb to hundreds of fb for Higgs boson masses up to several hundred $\mathrm{GeV}$.

\section{Future perspectives}

After two years of shutdown, the LHC has restarted its operations in spring 2015 and has delivered the first $13 \mathrm{TeV} p p$ collisions to both ATLAS and CMS. This second data-taking period (Run 2) should last until the end of 2018 and should deliver to both experiment about $100 \mathrm{fb}^{-1}$ of $p p$ collisions at $\sqrt{s}=13 \mathrm{TeV}$. On the longer term, the LHC Run 3 (2021-2023) is expected to 
provide about $200 \mathrm{fb}^{-1}$ of additional $p p$ collisions at the design energy of $\sqrt{s}=14 \mathrm{TeV}$, and the socalled high-luminosity phase of LHC (HL-LHC) should deliver about $3000 \mathrm{fb}^{-1}$ of $p p$ collisions at $14 \mathrm{TeV}$ by 2035.

Going from $\sqrt{s}=8 \mathrm{TeV}$ to $\sqrt{s}=13 \mathrm{TeV}$, the $\mathrm{SM}$ production cross sections for a Higgs boson with mass near $125 \mathrm{GeV}$ in Run 1 increase by factors ranging between $2(\mathrm{VH})$ and $3.9(t t H)$. The cross sections at $14 \mathrm{TeV}$ are slightly higher than the $13 \mathrm{TeV}$ ones. The background cross sections are also larger at $\sqrt{s}=13 \mathrm{TeV}$ than at $8 \mathrm{TeV}$, typically increasing two $(W, Z, \gamma \gamma)$ or three times $(t \bar{t})$.

In Run2, the increase of integrated luminosity (four times larger) and the larger signal cross sections due to the increase of the center-of-mass energy compared to Run 1 will lead to a sample that is approximately one order of magnitude larger than in Run 1 and will allow more precise studies of the Higgs boson sector. Such large dataset will allow more precise measurements of the Higgs boson couplings to bosons and to the heaviest fermions $(t, \tau)$ and of fiducial and differential cross-sections at $\sqrt{s}=13 \mathrm{TeV}$. Some exclusive production $(V H, t t H)$ and decay modes $(H \rightarrow b \bar{b})$ should be finally observed with significances larger than $5 \sigma$, and the Higgs boson coupling to $b$ quarks should be measured with $\mathscr{O}(10 \%)$ accuracy. More data will also shed light on a few excesses observed in Run $1(H \rightarrow \tau \mu, t t H)$, and the increase of the beam energy will significantly improve the sensitivity of BSM searches of heavy particles related to the Higgs sector, since the cross sections of processes taking place at energy scales of several hundred of $\mathrm{GeV}$ (or multi-TeV) increase by factors that are much larger (even by one order of magnitude or more) than those of the backgrounds.

Ultimately, the huge sample collected at the HL-LHC should lead to measurements of Higgs boson couplings to the few $\%$ level, to observe at more than $5 \sigma$ its couplings to second generation fermions (in $H \rightarrow \mu \mu$ ), to obtain a strong evidence of loop-induced rare decays like $H \rightarrow Z \gamma$, to set some constraints on the Higgs boson self-coupling [69, 70] and to perform many direct BSM searches with unprecedented experimental sensitivity.

\section{Conclusion}

The ATLAS and CMS experiments have discovered in July 2012 a new particle, with mass near $125 \mathrm{GeV}$, decaying to pairs of bosons $\left(\gamma \gamma, Z Z^{*}, W W^{*}\right)$. Using the $\sqrt{s}=7$ and $8 \mathrm{TeV} p p$ collision data delivered by the LHC during Run 1 (2010-2012), ATLAS has measured with an uncertainty at the level of $0.3 \%$ the mass of this resonance $\left(m_{H}=125.36 \mathrm{GeV}\right)$ and has investigated in great detail is other properties. All the results are consistent with the expectations for a SM Higgs boson of such a mass.

Evidence has been obtained for the decay of this particle to fermions $\left(\tau^{+} \tau^{-}\right)$and for production in vector-boson fusion, as well as in the dominant mode of gluon fusion; hints of production in associated modes ( $V H$ and $t t H$ ) and of decays to $b \bar{b}$ have been found. Spin and parity hypotheses alternative to the SM prediction $\left(J^{P}=0^{+}\right)$are disfavored at $>99.9 \% \mathrm{CL}$, and the measured upper limit on the total width $\left(\Gamma_{H}<22.7 \mathrm{MeV}\right)$ is consistent with the narrow width $(4.12 \mathrm{MeV})$ expected in the SM. The observed production cross sections and decay branching ratios of this particle, as well as its couplings to the weak bosons and to the heavier elementary fermions, are in agreement with the SM within uncertainties at the 10-20\% level. No evidence has been found of decays of 
this particle to final states that in the SM are either forbidden or suppressed to below the current experimental reach (like $H \rightarrow \mu \mu$ and $H \rightarrow Z \gamma$ ), nor strong indications of additional Higgs bosons have been found.

The new LHC data-taking period at $\sqrt{s}=13 \mathrm{TeV}$ (Run 2) that started in 2015 and the longer term LHC and ATLAS data-taking plans will allow a rich and diversified program of searches for deviations from the SM or for extra states in the scalar sector. After the Higgs discovery era, a new period of precision measurements has thus just started.

\section{References}

[1] ATLAS Collaboration, "The ATLAS Experiment at the CERN Large Hadron Collider," JINST 3 (2008) S08003.

[2] L. Evans and P. Bryant, "LHC Machine," JINST 3 (2008) S08001.

[3] S. L. Glashow, "Partial Symmetries of Weak Interactions," Nucl. Phys. 22 (1961) 579.

[4] S. Weinberg, "A Model of Leptons,” Phys. Rev. Lett. 19 (1967) 1264.

[5] A. Salam, "Weak and Electromagnetic Interactions," Conf. Proc. C 680519 (1968) 367.

[6] S. L. Glashow, J. Iliopoulos and L. Maiani, "Weak Interactions with Lepton-Hadron Symmetry," Phys. Rev. D 2 (1970) 1285.

[7] M. Gell-Mann and Y. Neeman, “The Eight Fold Way” (1964), Benjamin ed. (New York).

[8] P. W. Higgs, “Broken symmetries, massless particles and gauge fields," Phys. Lett. 12 (1964) 132.

[9] P. W. Higgs, "Broken Symmetries and the Masses of Gauge Bosons," Phys. Rev. Lett. 13 (1964) 508.

[10] G. S. Guralnik, C. R. Hagen and T. W. B. Kibble, "Global Conservation Laws and Massless Particles," Phys. Rev. Lett. 13 (1964) 585.

[11] F. Englert and R. Brout, "Broken Symmetry and the Mass of Gauge Vector Mesons," Phys. Rev. Lett. 13 (1964) 321.

[12] P. W. Higgs, "Spontaneous Symmetry Breakdown without Massless Bosons," Phys. Rev. 145 (1966) 1156.

[13] T. W. B. Kibble, "Symmetry breaking in nonAbelian gauge theories," Phys. Rev. 155, 1554 (1967).

[14] ATLAS Collaboration, "Observation of a new particle in the search for the Standard Model Higgs boson with the ATLAS detector at the LHC," Phys. Lett. B 716 (2012) 1 [arXiv:1207.7214 [hep-ex]].

[15] CMS Collaboration, "Observation of a new boson at a mass of $125 \mathrm{GeV}$ with the CMS experiment at the LHC," Phys. Lett. B 716 (2012) 30 [arXiv:1207.7235 [hep-ex]].

[16] ATLAS Collaboration, "Performance of the ATLAS Inner Detector Track and Vertex Reconstruction in the High Pile-Up LHC Environment", ATLAS-CONF-2012-042 (2012),

https://cds.cern.ch/record/1435196.

[17] ATLAS Collaboration, "Electron reconstruction and identification efficiency measurements with the ATLAS detector using the 2011 LHC proton-proton collision data," Eur. Phys. J. C 74 (2014) no.7, 2941 [arXiv:1404.2240 [hep-ex]].

[18] ATLAS Collaboration, "Electron efficiency measurements with the ATLAS detector using the 2012 LHC proton-proton collision data," ATLAS-CONF-2014-032 (2014),

https://cds.cern.ch/record/1706245. 
[19] ATLAS Collaboration, "Expected photon performance in the ATLAS experiment," ATL-PHYS-PUB-2011-007 (2011), https : / / cds . cern. ch/record/1345329.

[20] ATLAS Collaboration, "Measurements of the photon identification efficiency with the ATLAS detector using $4.9 \mathrm{fb}^{1}$ of $p p$ collision data collected in 2011," ATLAS-CONF-2012-123 (2012), https://cds.cern.ch/record/1473426

[21] ATLAS Collaboration, "Electron and photon energy calibration with the ATLAS detector using LHC Run 1 data,” Eur. Phys. J. C 74 (2014) no.10, 3071 [arXiv:1407.5063 [hep-ex]].

[22] ATLAS Collaboration, "Measurement of the muon reconstruction performance of the ATLAS detector using 2011 and 2012 LHC proton-proton collision data," Eur. Phys. J. C 74 (2014) no.11, 3130 [arXiv:1407.3935 [hep-ex]].

[23] M. Cacciari, G. P. Salam and G. Soyez, “The anti- $k_{t}$ jet clustering algorithm,” JHEP 0804 (2008) 063 [arXiv:0802.1189 [hep-ph]].

[24] ATLAS Collaboration, "Jet energy measurement and its systematic uncertainty in proton-proton collisions at $\sqrt{s}=7 \mathrm{TeV}$ with the ATLAS detector,” Eur. Phys. J. C 75 (2015) 17 [arXiv:1406.0076 [hep-ex]].

[25] ATLAS Collaboration, "Data-driven determination of the energy scale and resolution of jets reconstructed in the ATLAS calorimeters using dijet and multijet events at $\sqrt{\mathrm{s}}=8^{\prime \prime} \mathrm{TeV}$, ATLAS-CONF-2015-017 (2015), https : / / cds . cern . ch/record/2008678.

[26] ATLAS Collaboration, "Determination of the jet energy scale and resolution at ATLAS using $Z / \gamma$-jet events in data at $\sqrt{s}=8 \mathrm{TeV}$," ATLAS-CONF-2015-057 (2015), https: //cds.cern. ch/record/2059846.

[27] ATLAS Collaboration, "Performance of $b$-Jet Identification in the ATLAS Experiment," JINST 11 (2016) no.04, P04008 [arXiv:1512.01094 [hep-ex]].

[28] ATLAS Collaboration, "Performance of Missing Transverse Momentum Reconstruction in Proton-Proton Collisions at 7 TeV with ATLAS,” Eur. Phys. J. C 72 (2012) 1844 [arXiv:1108.5602 [hep-ex]].

[29] ATLAS Collaboration, "Performance of Missing Transverse Momentum Reconstruction in ATLAS studied in Proton-Proton Collisions recorded in 2012 at 8 TeV," ATLAS-CONF-2013-082 (2013), https: //cds.cern. ch/record/1570993.

[30] ATLAS Collaboration, "Identification and energy calibration of hadronically decaying tau leptons with the ATLAS experiment in $p p$ collisions at $\sqrt{s}=8$ TeV," Eur. Phys. J. C 75 (2015) no.7, 303 [arXiv:1412.7086 [hep-ex]].

[31] LHC Higgs Cross Section Working Group, "Handbook of LHC Higgs cross sections: 1. Inclusive observables", CERN-2011-002 (2011) [arXiv:1101.0593 [hep-ph]].

[32] LHC Higgs Cross Section Working Group, "Handbook of LHC Higgs cross sections: 2. Differential distributions”, CERN-2012-002 (2012) [arXiv:1201.3084 [hep-ph]].

[33] ATLAS and CMS Collaborations, "Procedure for the LHC Higgs boson search combination in Summer 2011”, ATL-PHYS-PUB-2011-011, CMS-NOTE-2011/005 (2011), https://cds.cern.ch/record/1379837

[34] A. L. Read, "Presentation of search results: The $C L_{s}$ technique", J. Phys. G: Nucl. Part. Phys 28 (2002) 2693. 
[35] M. Baak et al. [Gfitter Group Collaboration], "The global electroweak fit at NNLO and prospects for the LHC and ILC,” Eur. Phys. J. C 74 (2014) 3046 [arXiv:1407.3792 [hep-ph]].

[36] ATLAS Collaboration, "Measurement of the Higgs boson mass from the $H \rightarrow \gamma \gamma$ and $H \rightarrow Z Z^{*} \rightarrow 4 \ell$ channels with the ATLAS detector using $25 \mathrm{fb}^{-1}$ of $p p$ collision data," Phys. Rev. D 90 (2014) 052004 [arXiv:1406.3827 [hep-ex]].

[37] ATLAS and CMS Collaborations, "Combined Measurement of the Higgs Boson Mass in $p p$ Collisions at $\sqrt{s}=7$ and $8 \mathrm{TeV}$ with the ATLAS and CMS Experiments," Phys. Rev. Lett. 114 (2015) 191803 [arXiv:1503.07589 [hep-ex]].

[38] ATLAS Collaboration, "Measurements of the Higgs boson production and decay rates and coupling strengths using $p p$ collision data at $\sqrt{s}=7$ and $8 \mathrm{TeV}$ in the ATLAS experiment," Eur. Phys. J. C 76 (2016) no.1, 6 [arXiv:1507.04548 [hep-ex]].

[39] ATLAS Collaboration, "Measurement of Higgs boson production in the diphoton decay channel in $p p$ collisions at center-of-mass energies of 7 and $8 \mathrm{TeV}$ with the ATLAS detector," Phys. Rev. D 90 (2014), 112015 [arXiv:1408.7084 [hep-ex]].

[40] ATLAS Collaboration, "Search for $H \rightarrow \gamma \gamma$ produced in association with top quarks and constraints on the Yukawa coupling between the top quark and the Higgs boson using data taken at $7 \mathrm{TeV}$ and 8 TeV with the ATLAS detector,’ Phys. Lett. B 740 (2015) 222 [arXiv:1409.3122 [hep-ex]].

[41] ATLAS Collaboration, "Measurements of Higgs boson production and couplings in the four-lepton channel in $p p$ collisions at center-of-mass energies of 7 and $8 \mathrm{TeV}$ with the ATLAS detector," Phys. Rev. D 91 (2015) no.1, 012006 [arXiv:1408.5191 [hep-ex]].

[42] ATLAS Collaboration, "Search for the associated production of the Higgs boson with a top quark pair in multilepton final states with the ATLAS detector," Phys. Lett. B 749 (2015) 519 [arXiv:1506.05988 [hep-ex]].

[43] ATLAS Collaboration, "Observation and measurement of Higgs boson decays to $W W^{*}$ with the ATLAS detector,” Phys. Rev. D 92 (2015) no.1, 012006 [arXiv:1412.2641 [hep-ex]].

[44] ATLAS Collaboration, "Study of (W/Z)H production and Higgs boson couplings using $H \rightarrow W W^{*}$ decays with the ATLAS detector," JHEP 1508 (2015) 137 [arXiv:1506.06641 [hep-ex]].

[45] ATLAS Collaboration, "Evidence for the Higgs-boson Yukawa coupling to tau leptons with the ATLAS detector,” JHEP 1504 (2015) 117 [arXiv:1501.04943 [hep-ex]].

[46] ATLAS Collaboration, "Search for the $b \bar{b}$ decay of the Standard Model Higgs boson in associated $(W / Z) H$ production with the ATLAS detector," JHEP 1501 (2015) 069 [arXiv:1409.6212 [hep-ex]].

[47] ATLAS Collaboration, "Search for the Standard Model Higgs boson produced in association with top quarks and decaying into $b \bar{b}$ in $p p$ collisions at $\sqrt{s}=8 \mathrm{TeV}$ with the ATLAS detector," Eur. Phys. J. C 75 (2015) no.7, 349 [arXiv:1503.05066 [hep-ex]].

[48] ATLAS Collaboration, "Search for Higgs boson decays to a photon and a $Z$ boson in $p p$ collisions at $\sqrt{s}=7$ and $8 \mathrm{TeV}$ with the ATLAS detector,' Phys. Lett. B 732 (2014) 8 [arXiv:1402.3051 [hep-ex]].

[49] ATLAS Collaboration, "Search for the Standard Model Higgs boson decay to $\mu^{+} \mu^{-}$with the ATLAS detector," Phys. Lett. B 738 (2014) 68 [arXiv:1406.7663 [hep-ex]].

[50] CMS Collaboration, "Precise determination of the mass of the Higgs boson and tests of compatibility of its couplings with the standard model predictions using proton collisions at 7 and $8 \mathrm{TeV}$," Eur. Phys. J. C 75 (2015) no.5, 212 [arXiv:1412.8662 [hep-ex]]. 
[51] ATLAS and CMS Collaborations, "Measurements of the Higgs boson production and decay rates and constraints on its couplings from a combined ATLAS and CMS analysis of the LHC $p p$ collision data at $\sqrt{s}=7$ and 8 TeV," ATLAS-CONF-2015-044, CMS-PAS-HIG-15-002 (2015), https://cds.cern. ch/record/2052552.

[52] ATLAS Collaboration, "Measurements of the Total and Differential Higgs Boson Production Cross Sections Combining the $H \rightarrow \gamma \gamma$ and $H \rightarrow Z Z^{*} \rightarrow 4 \ell$ Decay Channels at $\sqrt{s}=8$ TeV with the ATLAS Detector," Phys. Rev. Lett. 115 (2015) 091801 [arXiv:1504.05833 [hep-ex]].

[53] ATLAS Collaboration, "Constraints on non-Standard Model Higgs boson interactions in an effective Lagrangian using differential cross sections measured in the $H \rightarrow \gamma \gamma$ decay channel at $\sqrt{s}=8 \mathrm{TeV}$ with the ATLAS detector,” Phys. Lett. B 753 (2016) 69 [arXiv:1508.02507 [hep-ex]].

[54] ATLAS Collaboration, "Constraints on the off-shell Higgs boson signal strength in the high-mass ZZ and $W W$ final states with the ATLAS detector,” Eur. Phys. J. C 75 (2015) no.7, 335 [arXiv:1503.01060 [hep-ex]].

[55] ATLAS Collaboration, "Evidence for the spin-0 nature of the Higgs boson using ATLAS data," Phys. Lett. B 726 (2013) 120 [arXiv:1307.1432 [hep-ex]].

[56] ATLAS Collaboration, "Study of the spin and parity of the Higgs boson in diboson decays with the ATLAS detector,” Eur. Phys. J. C 75 (2015) no.10, 476 Erratum: [Eur. Phys. J. C 76 (2016) no.3, 152] [arXiv:1506.05669 [hep-ex]].

[57] ATLAS Collaboration, "Search for lepton-flavour-violating $H \rightarrow \mu \tau$ decays of the Higgs boson with the ATLAS detector,” JHEP 1511 (2015) 211 [arXiv:1508.03372 [hep-ex]].

[58] ATLAS Collaboration, "Search for Invisible Decays of a Higgs Boson Produced in Association with a Z Boson in ATLAS,” Phys. Rev. Lett. 112 (2014) 201802 [arXiv:1402.3244 [hep-ex]].

[59] ATLAS Collaboration, "Search for invisible decays of the Higgs boson produced in association with a hadronically decaying vector boson in $p p$ collisions at $\sqrt{s}=8 \mathrm{TeV}$ with the ATLAS detector," Eur. Phys. J. C 75 (2015) no.7, 337 [arXiv:1504.04324 [hep-ex]].

[60] ATLAS Collaboration, "Search for invisible decays of a Higgs boson using vector-boson fusion in $p p$ collisions at $\sqrt{s}=8 \mathrm{TeV}$ with the ATLAS detector," JHEP 1601 (2016) 172 [arXiv:1508.07869 [hep-ex]].

[61] ATLAS Collaboration, "Constraints on new phenomena via Higgs boson couplings and invisible decays with the ATLAS detector,” JHEP 1511 (2015) 206 [arXiv:1509.00672 [hep-ex]].

[62] ATLAS Collaboration, "Search for Higgs and $Z$ Boson Decays to $J / \psi \gamma$ and $\Upsilon(n S) \gamma$ with the ATLAS Detector,” Phys. Rev. Lett. 114 (2015) no.12, 121801 [arXiv:1501.03276 [hep-ex]].

[63] ATLAS Collaboration, "Search for new light gauge bosons in Higgs boson decays to four-lepton final states in $p p$ collisions at $\sqrt{s}=8 \mathrm{TeV}$ with the ATLAS detector at the LHC," Phys. Rev. D 92 (2015) no.9, 092001 [arXiv:1505.07645 [hep-ex]].

[64] ATLAS Collaboration, "Search for a CP-odd Higgs boson decaying to $Z h$ in $p p$ collisions at $\sqrt{s}=8$ TeV with the ATLAS detector," Phys. Lett. B 744 (2015) 163 [arXiv:1502.04478 [hep-ex]].

[65] ATLAS Collaboration, "Search for an additional, heavy Higgs boson in the $H \rightarrow Z Z$ decay channel at $\sqrt{s}=8 \mathrm{TeV}$ in $p p$ collision data with the ATLAS detector," Eur. Phys. J. C 76 (2016) no.1, 45 [arXiv:1507.05930 [hep-ex]]. 
[66] ATLAS Collaboration, "Search for Scalar Diphoton Resonances in the Mass Range 65 - $600 \mathrm{GeV}$ with the ATLAS Detector in $p p$ Collision Data at $\sqrt{s}=8$ TeV," Phys. Rev. Lett. 113 (2014) no.17, 171801 [arXiv:1407.6583 [hep-ex]].

[67] ATLAS Collaboration, "Search for a Charged Higgs Boson Produced in the Vector-Boson Fusion Mode with Decay $H^{ \pm} \rightarrow W^{ \pm} Z$ using $p p$ Collisions at $\sqrt{s}=8 \mathrm{TeV}$ with the ATLAS Experiment," Phys. Rev. Lett. 114 (2015) no.23, 231801 [arXiv:1503.04233 [hep-ex]].

[68] ATLAS Collaboration, "Search for charged Higgs bosons decaying via $H^{ \pm} \rightarrow \tau^{ \pm} v$ in fully hadronic final states using $p p$ collision data at $\sqrt{s}=8 \mathrm{TeV}$ with the ATLAS detector," JHEP 1503 (2015) 088 [arXiv:1412.6663 [hep-ex]].

[69] ATLAS Collaboration, "Projections for measurements of Higgs boson cross sections, branching ratios and coupling parameters with the ATLAS detector at a HL-LHC", ATL-PHYS-PUB-2013-014 (2013), https: / / cds. cern. ch/record/1611186.

[70] ATLAS Collaboration, "Prospects for measuring Higgs pair production in the channel $H \rightarrow \gamma \gamma H \rightarrow b \bar{b}$ using the ATLAS detector at the HL-LHC", ATL-PHYS-PUB-2014-019 (2014), https://cds.cern.ch/record/1956733. 